\title{
The influence of radiative effects on the accretion onto stellar magnetospheres
}

\author{
I. A. Kryukov ${ }^{1,2}$, N. V. Pogorelov ${ }^{1,2}$, U. Anzer² , G. S. Bisnovatyi-Kogan ${ }^{3}$, and G. Börner ${ }^{2}$ \\ ${ }^{1}$ Institute for Problems in Mechanics, Russian Academy of Sciences, Vernadskii Avenue 101-1, 119526 Moscow, Russia \\ e-mail: kryukov@ipmnet.ru, pgrlv@ipmnet.ru \\ 2 Max-Planck-Institut für Astrophysik, Karl-Schwarzschild-Straße 1, 85741 Garching, Germany \\ e-mail: ula@mpa-garching.mpg.de, grb@mpa-garching.mpg.de \\ 3 Space Research Institute, Russian Academy of Sciences, Profsoyuznaya St. 84/32, 117810 Moscow, Russia \\ e-mail: gkogan@mx.iki.rssi.ru
}

Received 17 September 2002 / Accepted 10 February 2003

\begin{abstract}
The influence of radiative effects on the accretion onto stellar magnetospheres is investigated by performing global 2and 2.5-dimensional simulations on the basis of high-resolution numerical schemes with the application of irregular grids adapted to the shape of the magnetopause. The latter is represented by an impermeable, contracted dipole magnetic field surface with polar holes. Accreting matter is assumed to be optically thin. The physical mechanisms which are taken into account include cooling due to free-free and free-bound transitions, the Compton heating via X-ray scattering on electrons, and the inverse Compton cooling in the regions where the temperature of the matter becomes sufficiently large to be able to transfer part of its internal energy to photons. Depending on the determining parameters, both steady-state solutions with a system of discontinuities and unsteady flows with expanding shock waves can be obtained. It is shown that efficient cooling of the matter can substantially facilitate the penetration of the matter through the polar holes. The detailed consideration of the realistic radiative effects proved to be of great importance in our understanding of the accretion phenomenon, since they can substantially affect it both qualitatively and quantitatively.
\end{abstract}

Key words. accretion, accretion disks - radiative transfer - stars: neutron - shock waves - methods: numerical

\section{Introduction}

Accretion onto neutron stars and black holes is the main energy supply in galactic X-ray sources. The angular momentum captured by the X-ray star from the optical companion's wind with velocity $V_{\infty}$ and the binary period $P$ is proportional to $V_{\infty}^{-4} P^{-1}$ (Illarionov \& Sunyaev 1975). If $V_{\infty}$ is high or the binary components are widely separated, the angular momentum is not sufficient for the accretion disk formation at distances of the Alfvén radius where the magnetic pressure of the star is balanced by the dynamic pressure of infalling matter, and the accretion at large distances from the star becomes nearly spherically symmetric, see Illarionov \& Sunyaev (1975), Shapiro \& Lightman (1976), and Arons \& Lea (1980). This occurs, for example, in some X-ray sources with a massive companion star where long-periodic pulsars are observed (Börner et al. 1987; Nagase 1989; Bisnovatyi-Kogan 1991; Anzer \& Börner 1995).

Solutions for the spherically symmetric accretion of a polytropic flow onto nonmagnetized stars have been previously obtained both analytically (Bondi 1952) and numerically (Ruffert 1994; Ruffert \& Arnett 1994) for finite accretor sizes

Send offprint requests to: N. V. Pogorelov, e-mail: pgrlv@ipmnet.ru and polytropic indices $\gamma$ equal to or less than 5/3. The latter value obviously corresponds to the adiabatic flow of a fully ionized plasma. By decreasing $\gamma$ below this value one can somehow model cooling processes that inevitably occur in a hightemperature plasma. On the other hand, any heating process will effectively increase the polytropic index.

Self-similar spherically symmetric accretion in the gravitation field of a point mass was comprehensively investigated by Kazhdan \& Murzina (1994) for the case of adiabatic flows with a power-law distribution of density far from the accreting center. It was shown that the most general solution involves a shock wave expanding from the center with the distance changing in time like $R \sim t^{2 / 3}$. Its velocity is indefinite and can be fixed only by specifying the nature of the infall at the center. As noted in Paper I (Kryukov et al. 2000), this implies that the shock forms due to the presence of imaginary impermeable or partially permeable surfaces near the origin. Obviously, these surfaces are no longer imaginary if we solve problems that are not self-similar. This is the case when accretion onto a stellar magnetosphere is studied.

One can expect that radiative cooling of the infalling gas will substantially change the accretion pattern. On the other 
hand, heating by the stellar radiation can also dramatically affect it by decreasing the accretion rate $\dot{\mathcal{M}}$ (Shvartzman 1970).

Spherical accretion onto compact X-ray sources with preheating of matter was studied by Buff \& McCray (1974), Ostriker et al. (1976), Cowie et al. (1978, 1981), Bisnovatyi-Kogan \& Blinnikov (1980, further on cited as BKB), and Stellingwerf \& Buff (1982). It was shown that if the accretion flow in the presence of radiative effects has properties similar to the Bondi flow (the sonic temperature $T_{\mathrm{s}}$ is of the order of the recombination temperature and $\rho_{\mathrm{s}} \approx \rho_{\infty}$ ), then for accretion efficiencies $E>E_{\text {cr }}$ steady accretion becomes impossible, provided that $L>L_{\mathrm{cr}} \approx 10^{-3} \times 10^{-2} \times L_{\mathrm{Edd}}$, where $L_{\mathrm{Edd}}$ is the Eddington luminosity. Here the radiation of the star is assumed to be isotropic and the luminosity is defined by $L=E \dot{M} c^{2}$, where $c$ is the speed of light. BKB argued that for $E>E_{\text {cr }}$ the properties of the flow could be not Bondi-like, the density at the sonic point could be much greater for the same accretion rate, and the temperature at this point could be of the order of the Compton temperature $T_{\mathrm{C}}$. In this case the flow far from the accretor is nearly isothermal and of settling type. Thus, it was proven that there is no thermal limit for accretion. Krolik \& London (1983) showed that Bisnovatyi-Kogan \& Blinnikov's solution should be thermally unstable. However, this instability leads only to strong temperature fluctuations in a quasi-steady accretion flow (BKB; Krolik \& London 1983). Chang \& Ostriker (1985) also speculated that the flow can be stabilized by the presence of standing shocks.

It is worth noting that all the cited results assumed that accretors emit X-rays in a spherically symmetric way. One must always keep in mind, however, that if this radiation is restricted within some solid angle, buoyancy forces can cause pronounced outflows that will destroy the idealized accretion pattern (Illarionov \& Kompaneets 1990; Igumenshchev et al. 1993).

To analyze realistic astrophysical cases one would require the application of multidimensional high-resolution calculations (Kulikovskii et al. 2000). Bisnovatyi-Kogan \& Pogorelov (1997) investigated the accretion of slowly rotating matter onto a gravitating center. The angular momentum in those calculations had never been chosen large enough to obtain an accretion disk regime. Chen et al. (1997) dwelt on the accretion pattern with expanding shock waves for substantially faster rotating matter. Toropin et al. (1999) have recently presented the results of their magnetohydrodynamic (MHD) computation of quasi-spherical Bondi-like accretion onto a magnetic dipole and obtained several solutions with expanding shock waves. The penetration of matter through the surface of the magnetosphere in their calculations occurred due to a substantial, artificially introduced, resistivity of the plasma. This circumstance was noted in Paper I, where a somewhat different implementation of the accretion scenario was used (Arons \& Lea 1976). According to this scenario, the plasma flow is initially decelerated by the interaction with the stellar magnetic field, with cusps forming in the polar regions of the magnetosphere. Further on, as a result of the Rayleigh-Taylor (interchange) instability, clumps of plasma penetrate inside the magnetosphere and, threaded by the magnetic field, freely fall onto the poles along unperturbed magnetic field lines under the action of gravity. To simplify the problem, Paper I ignored the actual process of penetration and assumed instead that, on entering the magnetosphere, these clumps of plasma become homogenized within a layer adjacent to the inner side of the initially impermeable surface of the magnetosphere and soon after that one can again consider the gas flow in the continuum approximation. Thus, the final stage of the scenario was investigated, in which the flow occurred around some modified shape of the inner boundary that could be interpreted as an Alfvén surface possessing polar holes. It is worth emphasizing that the geometrical size of these holes is mainly determined by the width of the penetration layer. As noted in Paper I, this depth cannot be smaller than the size of plasma blobs penetrating inside the layer, which is $0.1 \div 0.2$ of the characteristic size of the magnetosphere. This agrees with the observational estimates of the angular size of polar hot spots, which is 0.1 radian for the dipole component of the magnetic field $\sim 5 \times 10^{10} \mathrm{G}$ (Sheffer et al. 1992; Baushev \& Bisnovatyi-Kogan 1999; Scott et al. 2000).

Thus, the boundary consists of an impermeable surface with polar holes. The magnetic field in this case is concentrated inside this boundary, and we can consider a purely hydrodynamic description of the flow. Although somewhat simplified, this model allows us to simulate the averaged picture of accretion. It is, however, important that the accreted plasma attains rather high temperatures and its treatment in the polytropic approximation is questionable. It will be shown in the main body of the paper that by taking into account sufficiently realistic cooling and heating terms in the energy equation and the radiation pressure term in the momentum equation, implying that the latter can be important if the luminosity of the star approaches the Eddington limit, one can obtain solutions for a physically acceptable range of parameters. These solutions involve additional discontinuities and/or layers of steep increase in temperature. By performing the parametric analysis, we have obtained some accretion regimes that admit sufficiently high accretion rates, which allow steady flows. There are also unsteady accretion regimes with the shock wave propagating away from the star.

In addition to the analysis of the spherically symmetric (at large distances from the star) accretion we analyze the influence of rotation of the accreting matter. Numerical results are compared with steady spherically symmetric solutions by BKB.

A few remarks should be added to justify the application of our accretion model that involves rather simplified boundary conditions. Actually they represent correct boundary conditions on an unknown Alfvén surface. Thus, our simplification is in specifying a certain shape for this boundary. These conditions contain basic features of the wind accretion in the case of an extended magnetosphere, that is, they act to stop the radially directed inflow and force the matter toward magnetic poles of a star. In reality, the flow deceleration takes place at the initially impermeable magnetosphere. At this stage the matter loses the velocity component normal to the magnetospheric surface. Penetrating inside, it acquires velocity mainly in a tangential direction. For this reason, we can shift the inner boundary, choosing the displacement on the basis of our ability 
to relate the polar hole radii to hot spot sizes. We study the accretion of a slowly rotating, nonmagnetized, and infinitely conductive medium onto stars possessing a strong dipole magnetic field. In this case the whole magnetic flux is accumulated inside the magnetosphere (a well-known cusp-type solution) and no magnetic torque remains. On the other hand, some flow details may be missing in this case. This is mainly due to the assumption of an ideal plasma flow. It is possible that this assumption will be invalid in the immediate vicinity of the magnetosphere. In this case, certain torque forces between the accreting plasma and the magnetosphere and frictional heating of shear flows will become important. However, as long as all rotational velocities remain small compared with the free-fall and Keplerian velocities these effects will only give minor corrections to the flow pattern obtained in this paper. For all these reasons, we are convinced that our model leads to an acceptable approximate description of a real accretion flow. Precise MHD calculations would be rather complicated and require the development of new physical and mathematical models accounting for plasma penetration into the magnetosphere. They will be the subject of a future investigation. The current paper emphasizes the importance of radiative effects and answers the question about the possibility of quasi-stationary accretion patterns for reasonably small polar holes.

The paper is structured as follows. In Sect. 2 we give the mathematical formulation of the problem and describe the parameters governing the accretion process. Section 3 discusses the peculiarities of the applied numerical technique. In Sect. 4 we present the results of the simulations of the accretion onto a gravitating center with and without rotation. Sections 5 and 6 deal with the accretion onto stellar magnetospheres. The results obtained are discussed in Sect. 7.

\section{Statement of the problem}

The arguments given in Introduction allow us to perform an investigation on the basis of the Euler gas dynamic equations including the source terms responsible for the gravitational attraction from the star, the radiative pressure, and the cooling and heating terms. Thus, the matter is assumed to be ideal and perfect, with the equation of state in the form

$\varepsilon=\frac{p}{(\gamma-1) \rho}$

where $\varepsilon, p, \rho$, and $\gamma=5 / 3$ are the specific internal energy, the pressure, the density, and the adiabatic index, respectively. The thermal equation of state is

$p=2 \rho \frac{k T}{m_{\mathrm{p}}}$,

where $T, k$, and $m_{\mathrm{p}}$ are the temperature, the Boltzmann constant, and the proton mass. The coefficient 2 is chosen to take into account the pressures of electrons and protons, the temperatures of which are assumed to be the same in the neutral on the average, hydrogen plasma.

The problem is solved in the axisymmetric statement. The symmetry axis coincides with the polar symmetry axis $0 z$ of the contracted magnetosphere (Fig. 1), whose shape is defined by the approximate formula (Elsner \& Lamb 1976)

$R_{\mathrm{m}}=R_{*} \exp \left(\frac{\cos 2 \phi}{2}\right) ; \quad \frac{\pi}{2}-\theta=\phi+\frac{1}{2} \sin 2 \phi$.

In the first quadrant we have $0 \leq \phi \leq \pi / 2$. The polar angle $\theta$ is counted off the $z$-axis. At the equator, $R_{\mathrm{m}}=R_{\mathrm{e}}=R_{*} \sqrt{e}$. The value $R_{*}$ is chosen as a length scale of the problem. The polar holes are circular and their radius $d$ is taken as a parameter of the problem. We use this simplified formula in the following calculations, since to a certain extent it adequately describes the shape of the magnetopause, which is contracted by the accreting plasma. The gravitating object is placed at the origin. The polar axis also represents the axis of the flow rotation, if the latter occurs.

The formulation of the problem implies that the mass flux rate at the spherical outer boundary $R=R_{0}$ is fixed,

$\dot{\mathcal{M}}=4 \pi R_{0}^{2} \rho_{0} U_{0}$.

Here and further on the subscript 0 refers to values at this boundary and $U_{0}$ is the velocity of the radial inflow.

The dimensional form of the system of governing equations in the coordinate system $(x, z)$ shown in Fig. 1 is the following:

$\frac{\partial x \boldsymbol{U}}{\partial t}+\frac{\partial x \boldsymbol{E}}{\partial x}+\frac{\partial x \boldsymbol{G}}{\partial z}+\boldsymbol{H}_{1}+x \boldsymbol{H}_{2}-x \boldsymbol{H}_{\mathrm{C}}+x \boldsymbol{H}_{\mathrm{cool}}=\mathbf{0}$,

where

$\boldsymbol{U}=[\rho, \rho u, \rho v x, \rho w, e]^{\mathrm{T}}$,

$\boldsymbol{E}=\left[\rho u, \rho u^{2}+p, \rho u v x, \rho u w,(e+p) u\right]^{\mathrm{T}}$,

$\boldsymbol{G}=\left[\rho w, \rho u w, \rho v w x, \rho w^{2}+p,(e+p) w\right]^{\mathrm{T}}$,

$\boldsymbol{H}_{1}=\left[0,-p-\rho v^{2}, 0,0,0\right]^{\mathrm{T}}$,

$\boldsymbol{H}_{2}=\left(1-\frac{L}{L_{\mathrm{Edd}}}\right) \frac{\rho G \mathcal{M}}{R^{2}}\left[\begin{array}{c}0 \\ \cos \theta \\ 0 \\ \sin \theta \\ u \sin \theta+w \cos \theta\end{array}\right]$,

$\boldsymbol{H}_{\mathrm{C}}=[0,0,0,0, H]^{\mathrm{T}}, \quad H=\frac{k \sigma_{\mathrm{T}} \dot{\mathcal{M}}}{\pi m_{\mathrm{p}} m_{\mathrm{e}}} E \frac{\rho\left(T_{\mathrm{C}}-T\right)}{R^{2}}$,

$\boldsymbol{H}_{\text {cool }}=[0,0,0,0, C]^{\mathrm{T}}, \quad C=n^{2} \Lambda(T)=\frac{\rho^{2}}{m_{\mathrm{p}}^{2}} \Lambda(T)$.

In these formulas $\boldsymbol{v}=[u, v, w]^{\mathrm{T}}$ is the velocity vector and $e=p /(\gamma-1)+\rho|\boldsymbol{v}|^{2} / 2$ is the total energy per unit volume. $\boldsymbol{H}_{1}$ is a geometrical source term that appears, since in the axisymmetric case we can exactly calculate the partial derivative $\partial / \partial y$ in the three-dimensional system of the Euler equations (the $y$ axis is perpendicular to the plane shown in Fig. 1). The given form of this term is valid in the half-plane $x \geq 0, y=0$. Note that the third equation in system (5) is written in the form of the angular momentum conservation law and has no source term. This allows one to ensure the exact conservation of this important physical quantity, which can be particularly important for 


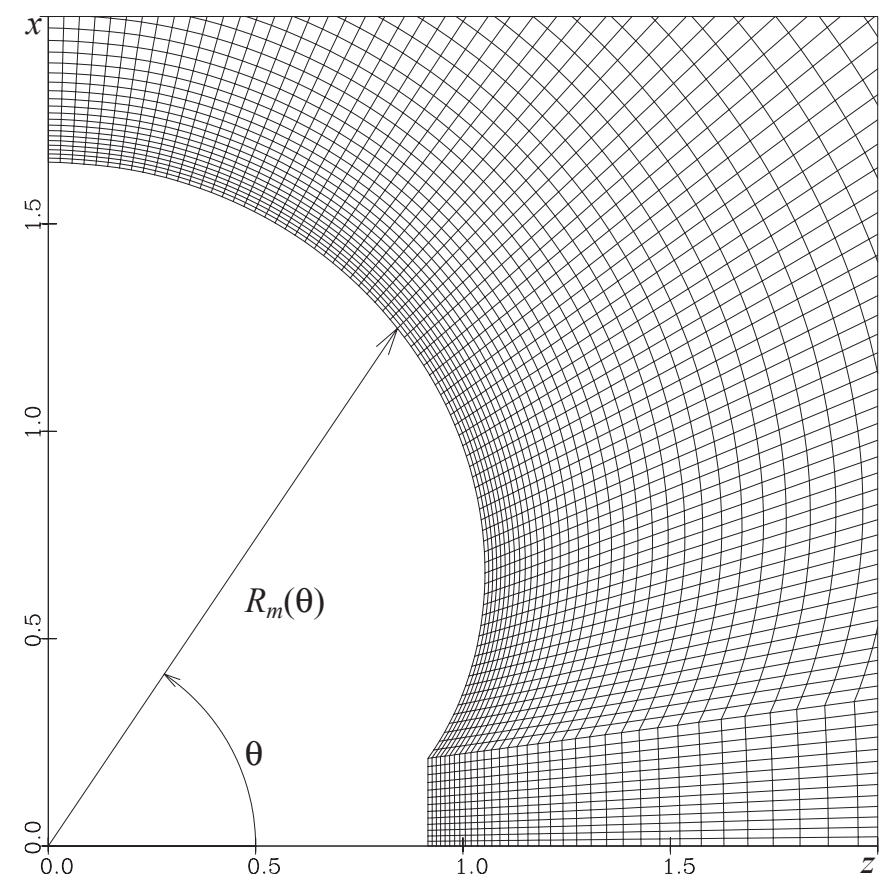

Fig. 1. Computational grid and the shape of the model magnetosphere.

modelling of flows with the specified axis of rotation (z-axis in our case). This form of the system has already been used by Molteni et al. (1999).

The vector $\boldsymbol{H}_{2}$ describes the action of gravity, which is modulated by the presence of the radiation pressure force in the momentum equations and its work in the energy equation. Here we assumed that the radiation flux is spatially isotropic. For this reason the total luminosity $L$ is connected with the spectrum averaged luminosity $\mathcal{L}$ per unit solid angle $\Omega$ by the formula

$L=\int_{4 \pi} \mathcal{L} \mathrm{d} \Omega=2 \pi \int_{0}^{\pi} \mathcal{L}(\theta) \sin \theta \mathrm{d} \theta=4 \pi \mathcal{L}$.

In formula (10), $G, \mathcal{M}$, and $R$ are the gravitational constant, the mass of the star, and the distance from the star to the current point of the computational region.

Formula (11) describes the heating/cooling due to the Compton effect (Levich \& Sunyaev 1971; see also Park 1990). In this formula $\sigma_{\mathrm{T}}, m_{\mathrm{e}}$, and $T_{\mathrm{C}}$ are the Thomson scattering cross-section, the mass of the electron, and the Compton temperature, respectively. The cooling term due to radiative losses is represented by Eq. (12), where $\Lambda(T)$ should represent the cooling function, which is usually calculated for a low-density, optically thin gas of some chosen cosmic element abundances (see, e.g., Raymond et al. 1976). We shall give the expression approximating this function later.

Let us normalize the quantities of density, velocity, pressure, and temperature by $\rho_{0}, U_{0}, \rho_{0} U_{0}^{2}$, and $T_{0}$, respectively. Since the length scale is $R_{*}$, the time scale becomes $R_{*} / U_{0}$. Thus, the dimensionless system acquires a form similar to
Eq. (5), where the structure of $\boldsymbol{U}, \boldsymbol{E}, \boldsymbol{G}$, and $\boldsymbol{H}_{1}$ remains unchanged. The other source terms become

$$
\boldsymbol{H}_{2}=\left(1-\frac{L}{L_{\text {Edd }}}\right) \frac{\rho S}{R^{2}}\left[\begin{array}{c}
0 \\
\cos \theta \\
0 \\
\sin \theta \\
u \sin \theta+w \cos \theta
\end{array}\right],
$$

$H=S_{\mathrm{C}} E \frac{\rho\left(T_{\mathrm{C}}-T\right)}{R^{2}}, \quad C=S_{\mathrm{b}} \rho^{2} \bar{\Lambda}(T)$.

The dimensional cooling function is normalized here by some characteristic value $[\Lambda]$.

Formulas (13)-(14) involve three additional dimensionless parameters:

$S=\frac{G \mathcal{M}}{R_{*} U_{0}^{2}}, \quad S_{\mathrm{C}}=\frac{T_{0}}{R_{*} U_{0}^{3}} \frac{k \sigma_{\mathrm{T}} \dot{\mathcal{M}}}{\pi m_{\mathrm{p}} m_{\mathrm{e}}}, \quad S_{\mathrm{b}}=\frac{R_{*} \rho_{0}[\Lambda]}{U_{0}^{3} m_{\mathrm{p}}^{2}}$.

The equation of state (1) preserves its form, whereas the dimensionless thermal equation of state becomes

$p=\frac{\rho T}{\gamma M_{0}^{2}}, \quad M_{0}=\frac{U_{0}^{2}}{a_{0}^{2}}, \quad a_{0}^{2}=\frac{\gamma p_{0}}{\rho_{0}}$

where $M_{0}$ and $a_{0}$ are the Mach number and the speed of sound at the outer boundary.

We assume that the inflow at $R=R_{0}$ is either supersonic or sonic. Thus, all quantities at the outer boundary should be specified. In cases when the matter has angular momentum, it is convenient to introduce an additional dimensionless parameter $S_{\mathrm{r}}=G \mathcal{M} / \omega_{*}^{2} R_{*}^{3}$ that represents the squared ratio between the Keplerian velocity $U_{\mathrm{K}}=\left(G \mathcal{M} / R_{*}\right)^{1 / 2}$ at $R=R_{*}$ and the velocity of rotation $v_{*}=\omega_{*} R_{*}$, where $\omega_{*}$ is the angular velocity that can be attained at the distance $R_{*}$ from the axis of rotation under the assumption of a constant specific angular momentum distribution.

If the flow is spherically symmetric, then we have the following conditions at the outer boundary $R=R_{0}$ :

$\rho_{0}=1, U_{0}=-1, p_{0}=\frac{1}{\gamma M_{0}^{2}}, v_{0}=0, W_{0}=0$,

where $W_{0}$ is the $\theta$-component of the velocity vector. Once the parameter $S$ is chosen, we know the dimensional velocity at the outer boundary. Thus, by specifying in (15) the dimensional temperature and mass flux rate (4) we determine all dimensionless parameters of the problem.

To specify the boundary conditions in the case of rotating matter, we choose the dimensionless $R_{0}$ and introduce the following distribution of the dimensionless, in terms of $\omega_{*}$, angular velocity along the outer boundary:

$$
\begin{cases}\omega=\frac{1}{x^{2}}=\frac{1}{R_{0}^{2} \sin \theta} & \text { if } x>20, \\ \omega=0.0125 & \text { if } x \leq 20 .\end{cases}
$$

The intervals correspond to constant specific angular momentum and constant angular velocity distributions, respectively. Since we normalized the angular velocity by $\omega_{*}$, the formula for the $y$-component $v$ (normal to the plane of Fig. 1) of the 
velocity vector $\boldsymbol{v}$ should be calculated by the formula $v=$ $\omega x /\left(S_{\mathrm{r}} / S\right)^{1 / 2}$.

Because of the symmetry of the problem, it clearly suffices to seek the solution only in the first quadrant of the Cartesian coordinate system plane. The conditions on the inner boundary of the computational region are the following: nonpenetration is adopted in the angle interval $\theta_{*}<\theta<\pi / 2$ and absorbing boundary conditions are used for $0 \leq \theta \leq \theta_{*}$ (Pogorelov \& Semenov 1997), where $\theta_{*}$ corresponds to the edge of the polar hole.

\section{Numerical method}

The numerical solution of system (5) is performed with the help of the hydrodynamic package MHD-E-NS2D, developed for simulation of compressible gas and plasma flows governed by ideal magnetohydrodynamic (MHD) and purely gas dynamic equations and the Navier-Stokes equations (Ivanov \& Kryukov 1996; Pogorelov \& Matsuda 1998; Ivanov et al. 1999). The code is substantially based on numerical schemes that ensure high (second or third) order of accuracy and preserve the monotonicity of functions at discontinuities. Most of the incorporated methods can be classified as Godunov schemes and can be attributed to TVD (total variation diminishing), ENO (essentially nonoscillatory) or WENO (weighted ENO) classes, depending on the implementation of the code modules. To determine inviscid fluxes through the computational cell interfaces, either the exact or some of the approximate solutions to the Riemann problem of the disintegration of an arbitrary discontinuity are used (Kulikovskii et al. 2000). The methods are implemented on both stationary and moving structured adaptive grids. The numerical results presented below were obtained using substantially twodimensional reconstruction procedures for determining the values of the vector $\boldsymbol{U}$ on the right- and left-hand sides of the computational cell interfaces. The time integration is performed by third- or higher-order Runge-Kutta methods, whose application allows one to improve the overall stability of the numerical method.

\section{Accretion onto a gravitating center}

BKB obtained a numerical solution of the stationary gas dynamic equations for spherically symmetric accretion in the case of an optically thin flow with X-ray preheating. Of all the cooling mechanisms involved in the function $\Lambda(T)$ only bremsstrahlung was taken into account. The dimensional cooling term in Eq. (5) in this case takes the form $C=4.38 \times$ $10^{20} \rho^{2} T^{1 / 2} \mathrm{erg} \mathrm{cm}^{-3} \mathrm{~s}^{-1}$. The Compton temperature was chosen to be $T_{\mathrm{C}}=2.9 \times 10^{7} \mathrm{~K}$. To test the performance of the algorithm and the possibility of the development of instabilities, we solve the same problem on the basis of nonstationary equations, seeking steady-state solutions. As boundary conditions at $R=R_{0}$, we use the parameters at the sonic point of the flow determined by BKB. Two different cases are considered. The ratio $L / L_{\text {Edd }}$ and the efficiency $E$ of accretion are adopted to be 0.1 and 0.1 in the first case and 0.01 and 0.01 in the second one. Both cases correspond to the same accretion rate equal to

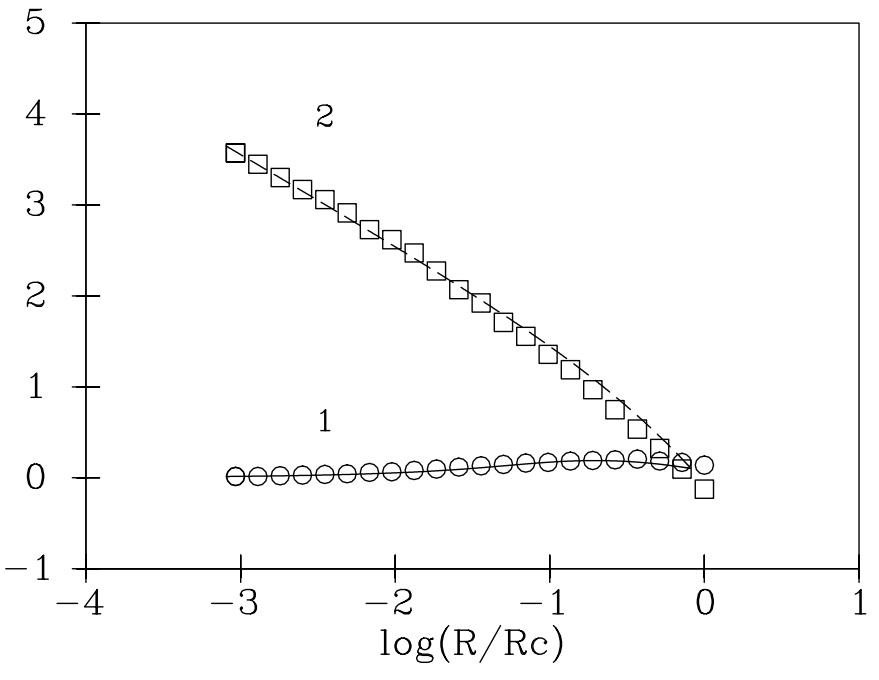

Fig. 2. Distributions of $\log \left(T / T_{\mathrm{C}}\right)$ (curve 1$)$ and $\log \left(U / U_{\mathrm{C}}\right.$ ) (curve 2) for the model BB1. Circles and squares refer to the results of BKB.

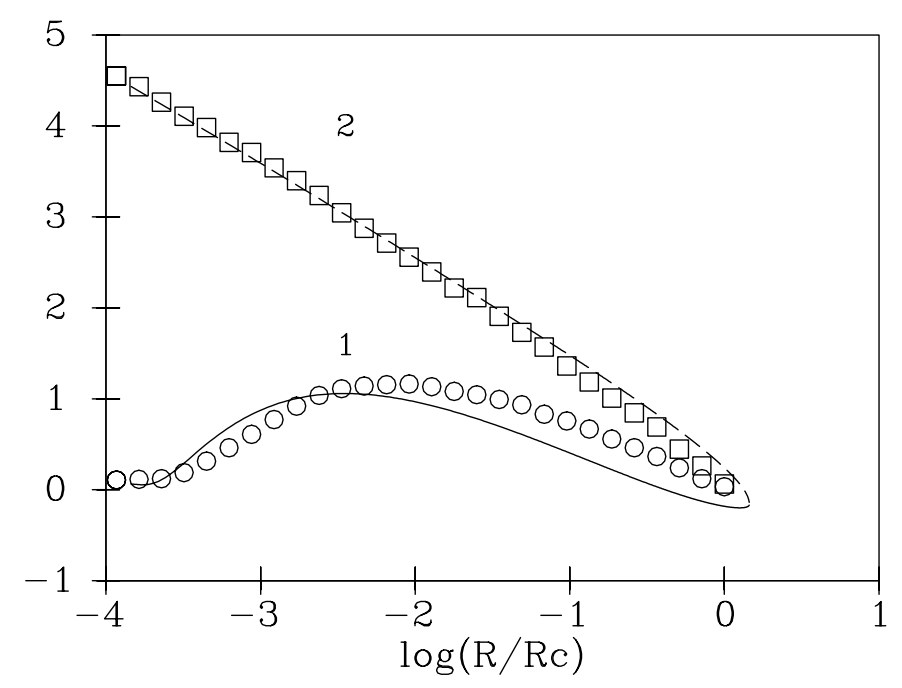

Fig. 3. Same as in Fig. 2, but for the model BB2.

$2.66 \times 10^{-9} \mathcal{M}_{\odot} / \mathrm{yr}$. We also assume that the mass of the accreting object is equal to the solar mass, that is, $\mathcal{M}=\mathcal{M}_{\odot}$. The radius $R_{*}$ of the inner boundary in our calculation of the accretion onto a gravitation center is related to the position $R=R_{0}$ of the before-mentioned sonic point. Since we assume that in the first case $R_{0}=10^{3} R_{*}$, we have $R_{*}=8.14 \times 10^{6} \mathrm{~cm}$. In the second case $R_{0}=10^{4} R_{*}$, thus giving $R_{*}=1.45 \times 10^{6} \mathrm{~cm}$. It is easy to calculate the parameter $S$ in these cases. It is equal to 1930 and $2 \times 10^{4}$, respectively. The temperature at the outer boundary is, respectively, $T_{0}=1.27 T_{\mathrm{C}}=3.683 \times 10^{7} \mathrm{~K}$ and $T_{0}=0.688 T_{\mathrm{C}}=2 \times 10^{7} \mathrm{~K}$.

The comparison of our numerical results (denoted as BB1 and BB2) with those of BKB are presented in Figs. 2 and 3, respectively. Here solid and dashed lines show the calculated radial distributions of $\log \left(T / T_{\mathrm{C}}\right)$ and $\log \left(U^{2} / U_{\mathrm{C}}^{2}\right)$, respectively, as functions of $\log \left(R / R_{\mathrm{C}}\right)$. The circles and squares correspond to the results of $\mathrm{BKB}$. Here $R_{\mathrm{C}}=G \mathcal{M} / 2 U_{\mathrm{C}}^{2}$ and $U_{\mathrm{C}}^{2}=\gamma k T_{\mathrm{C}} / \mu m_{\mathrm{p}}$, where $\mu=0.608$ is the mean molecular 


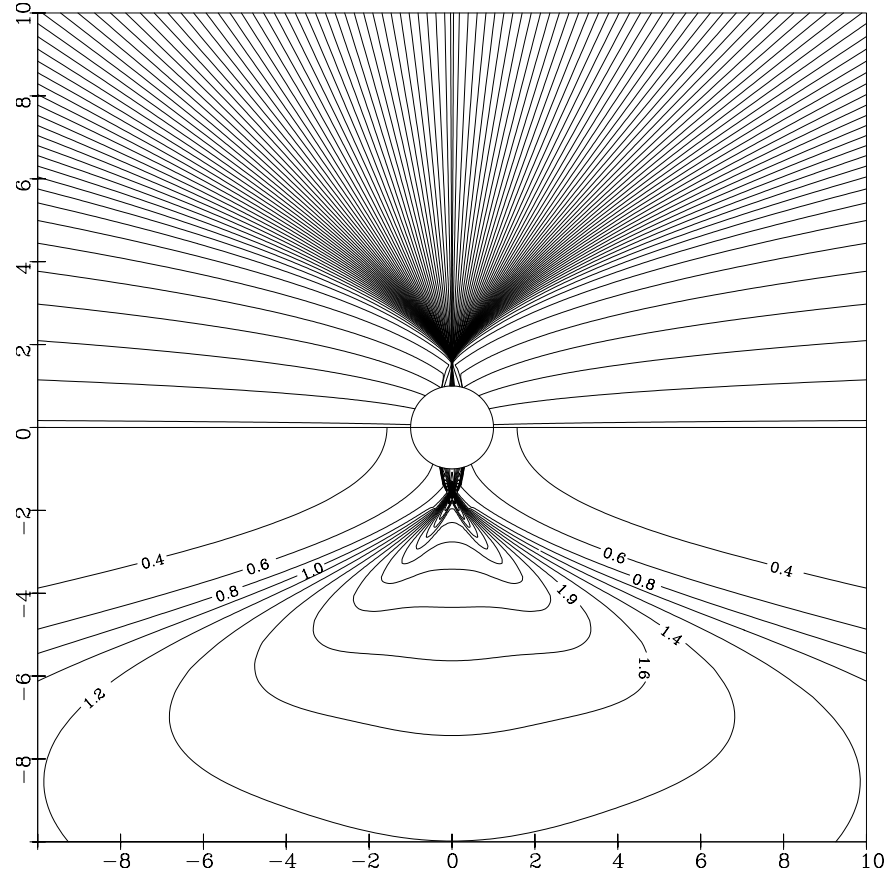

Fig. 4. The streamlines (above the symmetry axis) and the density logarithm distribution for the accretion of rotating matter onto a gravitating center.

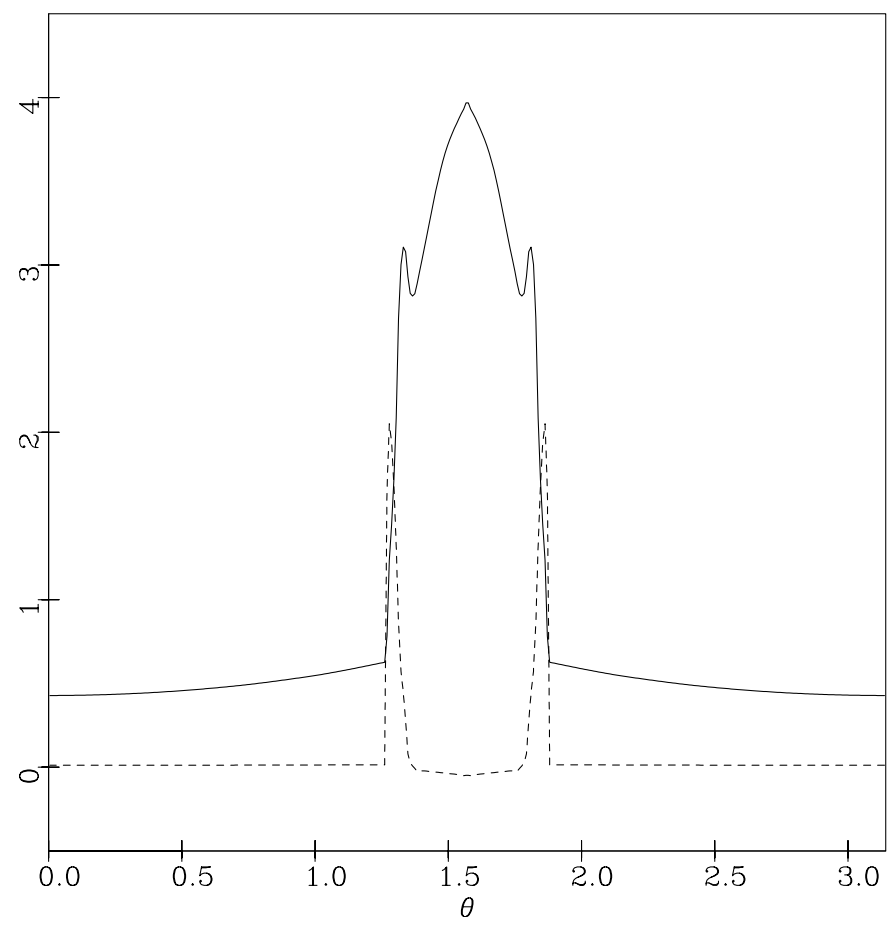

Fig. 5. Distributions of the density logarithm (solid line) and $\log \left(T / T_{\mathrm{C}}\right)$ along the inner boundary for the accretion of rotating matter onto a gravitating center.

weight used in BKB. The agreement of the results is quite satisfactory.

Consider now a similar case involving rotation of the accreting matter. As boundary conditions we choose the first of the previously considered BKB solutions, where temperatures are so high that only the bremsstrahlung branch of the cooling function is present. The angular velocity at the outer boundary is specified by the procedure described in Sect. 2. The value of the dimensionless parameter $S_{\mathrm{r}}$, responsible for the intensity of rotation, is set equal to 1.5. This means that for the conservation of the specific angular momentum the linear velocity of rotation at the equatorial point of the inner boundary will be $\sqrt{1.5}$ times less than the Keplerian velocity. This velocity is not sufficient to obtain an accretion disk. However, as seen from Fig. 4, a thin disk-like structure starts forming in the equatorial region well ahead of the corresponding point on the inner boundary. In this figure we show the streamlines (above the symmetry axis) and the isolines of the density logarithms. As a result of the action of the centrifugal force, most of the streamlines concentrate near the equatorial plane. The poloidal velocity component acquires rather high values, and when two branches of the flow meet at the equatorial plane they are decelerated at the shocks located on both sides of the disk. As the intensity of these shocks is very large, the temperature behind them greatly increases, giving rise to intense Compton cooling. This inevitably results in a subsequent decrease of the gas temperature, which is seen in Fig. 5 showing the logarithmic distributions of the gas temperature divided by the Compton temperature (dashed line) and density (solid line) with respect to the angle $\theta$ along the inner circular boundary. For this reason, the density and pressure first stop growing and even slightly decrease. As the temperature is nearly constant in the main body of the disk, the density increases again creating a high-density core of the disk. We found that the temperature in this core drops slightly below the Compton temperature on the inner boundary. This seems to be necessary to compensate additional hydrodynamic cooling of the compressed matter in the disk with the crosssection increasing towards the star. Note that maximum density and pressure values are reached at the position of minimum thickness. The results obtained are substantially different from those corresponding to the polytropic case presented in Paper I. They might reveal the dynamic scenario of the formation of accretion disks from quasi-radial flows possessing small angular momenta at large distances from the star.

\section{Quasi-spherical accretion onto the magnetosphere}

In this section we first present some results of the numerical simulation of the quasispherical accretion onto the model magnetosphere taking the parameter distribution at the outer boundary from the paper BKB, as in the previous section. We shall refer to these two variants as Var1 and Var2. The radii of the polar holes are chosen to be equal to 0.1 and 0.3 , respectively. In contrast to the polytropic case previously studied in Paper I, where it sufficed to specify at the outer boundary only the Mach number $M_{0}$ and the parameter $S$, we now need to know the dimensional values of the temperature $T_{0}$, the mass of the star $\mathcal{M}$, and the characteristic size $R_{*}$ of the magnetosphere. Then, by specifying the parameter $S$ and the accretion rate $\dot{\mathcal{M}}$ we find the Mach number and all the remaining dimensionless parameters occurring in the source terms of the governing system. Recall that in Var1 and Var2 we put $\mathcal{M}=\mathcal{M}_{\odot}$. To specify $R_{*}$ we use the following approximate formula, which is readily 


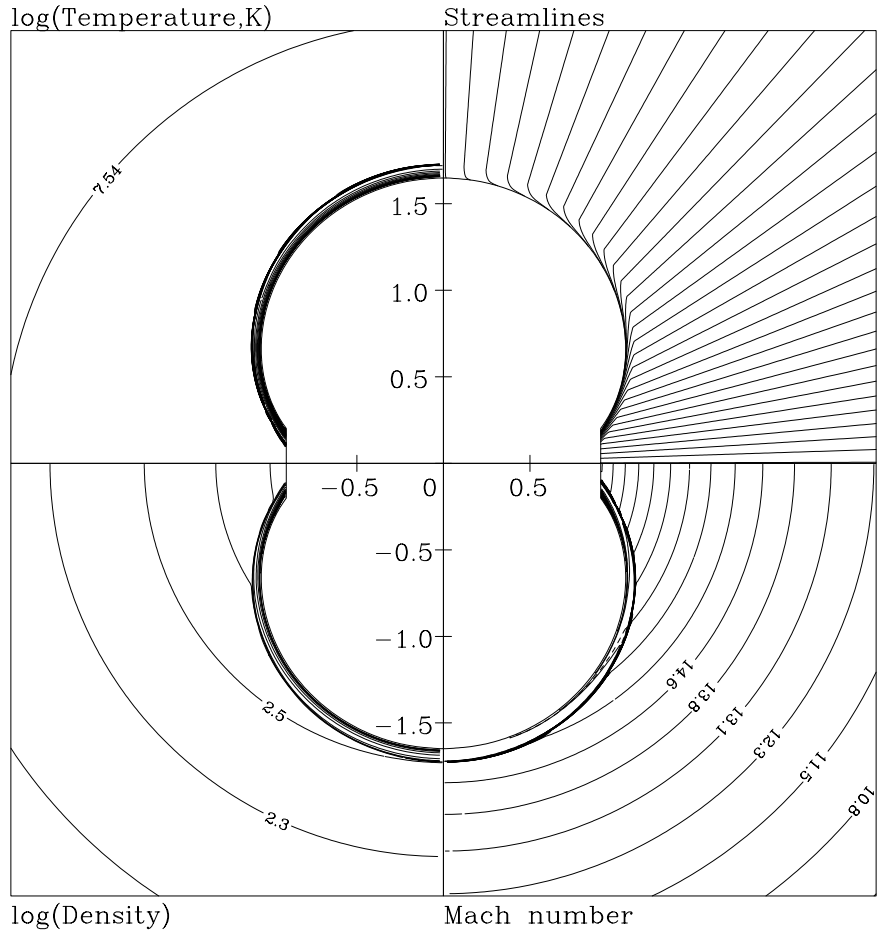

Fig. 6. Accretion pattern for the model Var1.

obtained by equating the dynamic pressure of the free falling gas to the magnetic pressure of the star at the equatorial stagnation point of the magnetosphere (Lipunov 1992):

$R_{*}=\left(\frac{B_{\mathrm{st}}^{2} R_{\mathrm{st}}^{6}}{\dot{\mathcal{M}} \sqrt{2 G M}}\right)^{2 / 7}$

where $R_{\mathrm{st}}$ and $B_{\mathrm{st}}$ are the radius of the star and the value of the dipolar magnetic field of the star at its poles. Further on we assume that $R_{\mathrm{st}}=10^{6} \mathrm{~cm}$. For Var1 and Var2 we set $B_{\mathrm{st}}=10^{11} \mathrm{G}$. This choice is justified by the fact that in these two variants the outer size of the computational region is fixed by the location of the sonic point in BKB's solution and we would not like this point to lie very close to the surface of the magnetosphere. Under these assumptions we obtain $R_{0}=109 R_{*} \approx$ $8.14 \times 10^{9} \mathrm{~cm}(\operatorname{Var} 1)$ and $R_{0}=193 R_{*} \approx 1.45 \times 10^{10} \mathrm{~cm}(\operatorname{Var} 2)$.

In Figs. 6 and 7 we give the distributions of different quantities in the computational region. In the first quadrant of the Cartesian coordinate system we show the streamlines. In the second quadrant, the isolines of the dimensional temperature logarithms are presented. The third quadrant contains the contours of constant density logarithms. Here we are usually able to see various discontinuities that are likely to occur when the flow is decelerated by the impermeable portion of the magnetospheric surface. In the fourth quadrant we show the contours of constant Mach numbers. Dotted lines represent the Compton temperature and the level $M=1$, respectively.

In Figs. 8 and 9 several one-dimensional plots are given. They represent the logarithms of the temperature (a), radial velocity (b), density (c), and Mach number (d) as functions of $\log R$. Solid and dash-and-dot lines correspond to the

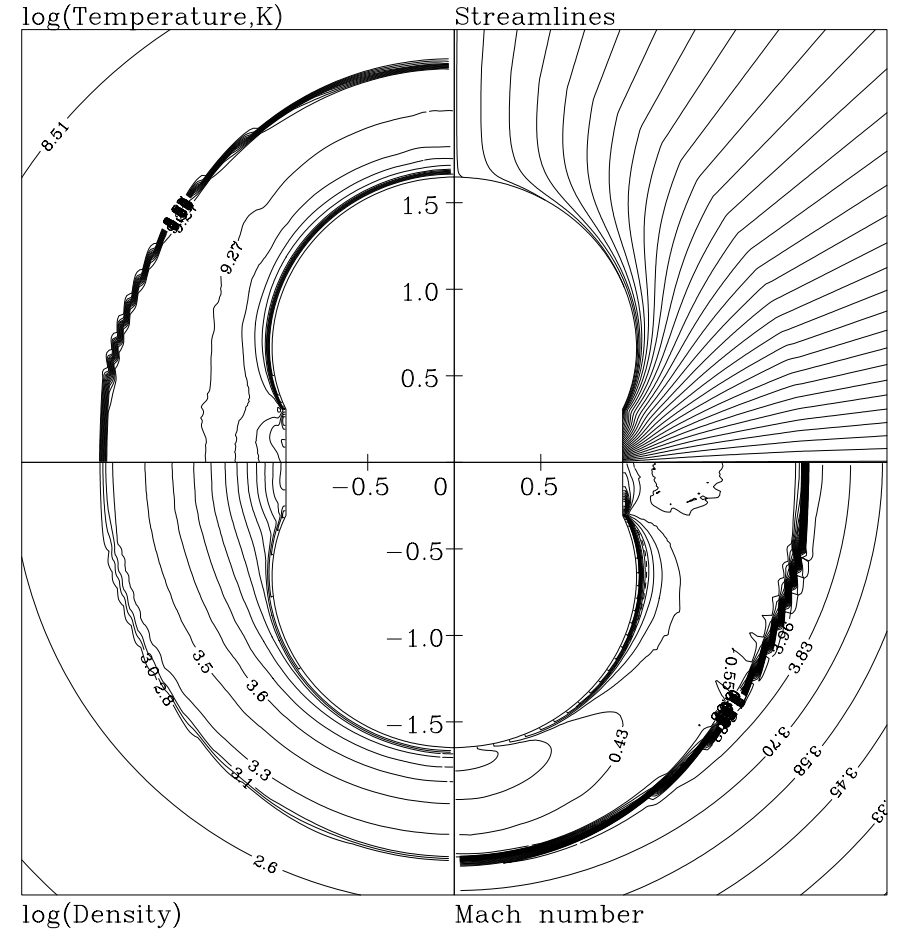

Fig. 7. Accretion pattern for the model Var2.

directions $\theta=\pi / 2$ and $\theta=0$, respectively (see Fig. 1). Dashed lines show the free-fall velocity contour, the Compton temperature, and the $M=1$ line.

In the adopted model, the flow is essentially a combination of supersonic blunt body and nozzle flows. For this reason a bow shock forms in front of the impermeable surface of the magnetosphere. This shock decelerates the accreting matter to subsonic velocities. It is clear that at $R=R_{\mathrm{e}}, \theta=\pi / 2$ we have a stagnation point. In addition, the shape of the magnetosphere is such that the radially oriented gravitational force has a component directed along this surface towards the poles. For this reason highly compressed gas moves to the poles and falls onto the stellar surface through open magnetic field lines. As seen from Fig. 6, there also exists a portion of the initially spherically symmetric flow that can be accreted directly through the polar holes without preliminary compression (Var1). On the other hand, for another set of parameters (Var2) this flow interacts with the stream of the gas in the shock layer, and decelerates to subsonic velocity forming another shock. These two shocks produce a cocoon of highly compressed hot gas around the magnetospheric surface. The temperature in this case remains substantially higher than the Compton temperature, since the inverse Compton effect cannot overcome gas dynamic heating due to the lower value of the accretion efficiency in Var2 (see Fig. 9). In Var1 the shock layer is very thin due to high values of the Mach number ahead of the shock and high compressibility of the gas inside it. The latter can be explained by the rapid cooling of the gas.

As seen from Figs. 8 and 9, the essential difference between Var1 and Var2 is in the value of temperature at the outer boundary. In the first case it is higher than the Compton temperature and both bremsstrahlung and the inverse Compton effect act 

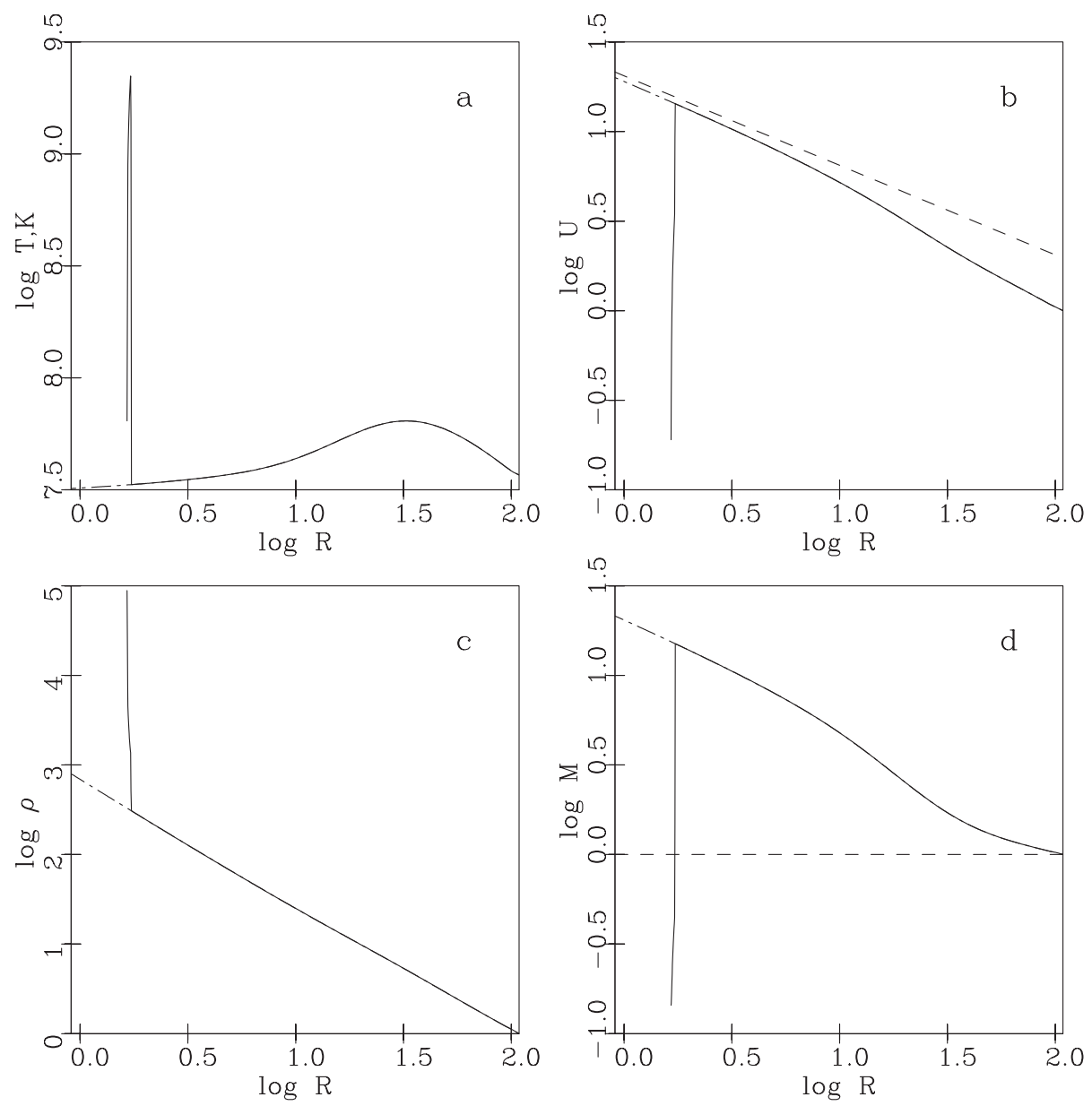

Fig. 8. Radial distributions of temperature a), radial velocity component b), density c), and Mach number d) along the $x$ - (solid lines) and $z$-axes (dash-and-dot lines). Dashed lines in figures b) and c) show the free-fall velocity and the $M=1$ value (Var1).

to cool the accreting matter. In the immediate vicinity of the outer boundary they cannot compete, however, with the adiabatic heating of the matter attracted by the stellar gravity. As the gas moves further towards the star the Compton cooling increases and the temperature ahead of the bow shock approaches the Compton temperature. As seen from the Mach number isolines, the shock is very strong. The temperature goes up while crossing it, although immediately decreases inside the shock layer due to the enormous influence of the inverse Compton effect. Along the polar axis, just a slight excess over the Compton temperature suffices to compensate adiabatic heating and the flow is nearly isothermal. The radial velocity along the polar axis asymptotically approaches the free-fall velocity, and the flow enters the hole at a supersonic speed. On the contrary, in Var2 the gas is heated monotonically to high temperatures all its way to the bow shock. The Mach number ahead of it remains moderately high and the temperature increase through it is substantially lower than in Var1. As was previously mentioned, this can be explained by the lower level of the stellar luminosity $(E=0.01)$ and weaker Compton cooling.

The consideration of these two accretion regimes confirms the results of Paper I in the respect that cooling of the accreting matter is favorable for accretion. If the gas temperature at the outer boundary is close to the Compton temperature, the Compton heating is either absent or disappears as the gas approaches the accretor. Then, if the accretion efficiency is sufficiently high, as it is in Var1, cooling processes become dominant, and the prescribed amount of the mass flux can easily be accreted even through very narrow holes. Preheating generally results in higher bow shock stand-off distances, provided that a steady-state solution can be obtained. Otherwise, solutions with an outward-expanding shock are realized, similarly to the case considered by Toropin et al. (1999).

Consider now different determining parameters and more realistic cooling functions. There are several available in the literature (Cox \& Tucker 1969; Buff \& McCray 1974; Raymond et al. 1976; Stellingwerf \& Buff 1982; Cowie et al. 1981; Bond et al. 1984; Nobili et al. 1991; Sutherland \& Dopita 1993; Hutchings \& Thomas 2000). The difference among the functions is not only in different approximations of available calculated data. The cooling function itself depends on the cosmic abundances of elements and their degree of ionization. In this paper we use the function by Cowie et al. (1981), which approximates the data by Raymond et al. (1976):

$$
\Lambda(T)= \begin{cases}0 & \text { for } T<10^{4} \mathrm{~K} \\ 1.1 \times 10^{-24} T^{0.55} & \text { for } 10^{4} \mathrm{~K} \leq T<10^{5} \mathrm{~K} \\ 6.2 \times 10^{-19} T^{-0.6} & \text { for } 10^{5} \mathrm{~K} \leq T<4 \times 10^{7} \mathrm{~K} \\ 2.7 \times 10^{-27} T^{0.5} & \text { for } T \geq 4 \times 10^{7} \mathrm{~K} .\end{cases}
$$

As noted by Buff \& McCray (1974), if stellar radiation substantially increases the ionization degree of trace elements by the photoionization process, the radiative cooling rate can be lower than in the purely collisional case. For the sake of comparison, 

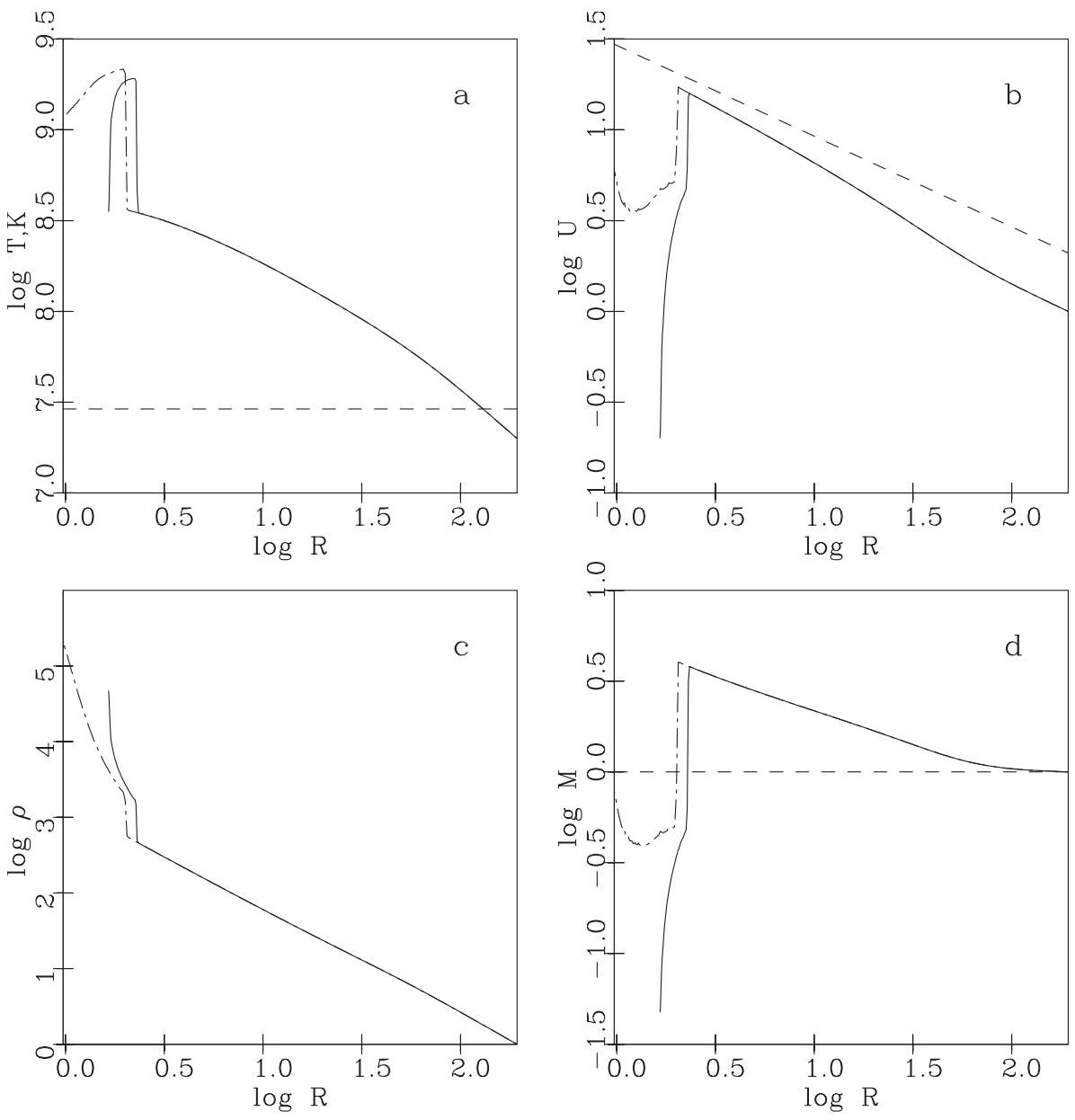

Fig. 9. Radial distributions of temperature a), radial velocity component b), density c), and Mach number d) along the $x$ (solid lines) and $z$-axes (dash-and-dot lines). Dashed lines in figures a), b), and c) show the Compton temperature value, the free-fall velocity, and the $M=1$ value (Var2).

we shall also present the results of calculations on the basis of an appropriate cooling function (Stellingwerf \& Buff 1982):

$$
\begin{aligned}
\Lambda(T)= & \left(\left[2.4 \times 10^{-27} T^{1 / 2}+6 \times 10^{-22} T^{-1 / 2}\right]^{-1}\right. \\
& \left.+2.5 \times 10^{75} T^{-12}\right)^{-1} .
\end{aligned}
$$

Both cooling functions in logarithmic scale are shown in Fig. 10.

In Figs. 11 and 14 results are shown that correspond to the following set of parameters: $\mathcal{M}=1.4 \mathcal{M}_{\odot}, \dot{\mathcal{M}}=10^{-8} \mathcal{M}_{\odot} / \mathrm{yr}$, $R_{*}=1.83 \times 10^{8} \mathrm{~cm}, R_{0}=100 R_{*}, S=200, T_{0}=10^{4}$, and $E=0.1$ (Var3). In Figs. 12 and 15 we present the distributions of the same functions for the case with $\mathcal{M}=1.4 \mathcal{M}_{\odot}$, $\dot{\mathcal{M}}=10^{-9} \mathcal{M}_{\odot} / \mathrm{yr}, R_{*}=3.52 \times 10^{8} \mathrm{~cm}, R_{0}=100 R_{*}, S=200$, $T_{0}=10^{4}$, and $E=0.1$ (Var4). As noted earlier, the characteristic size of the magnetosphere $R_{*}$ is evaluated on the basis of the value of $\dot{\mathcal{M}}$ for prescribed values of the radius and dipole magnetic field of the star $\left(R_{\mathrm{st}}=10^{6} \mathrm{~cm}\right.$ and $B_{\mathrm{st}}=10^{12} \mathrm{G}$ for both variants). The Compton temperature and the adiabatic heat ratio are equal to $3 \times 10^{7} \mathrm{~K}$ and $5 / 3$, respectively. The cooling function is that of Cowie et al. (1981). The chosen set of data shows that the velocity at the outer boundary is equal to 0.5 of the free-fall velocity $U_{\mathrm{ff}}=\sqrt{2 G \mathcal{M} / R_{0}}$. The Mach numbers appear to be very high in both cases: $M_{0} \approx 43$ (Var3) and $M_{0} \approx 31$ (Var4). Figures 13 and 16 correspond to the same

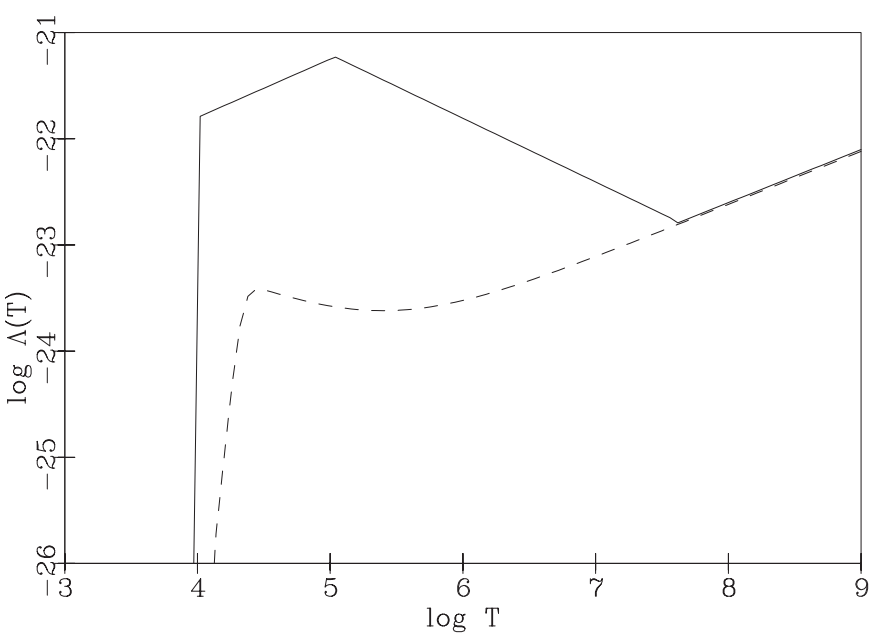

Fig. 10. The cooling functions of Cowie et al. (1981) and Stellinwerf $\&$ Buff (1982) shown by the solid and dashed lines, respectively.

set of parameters as in Var4, but with the use of the cooling function by Stellingwerf \& Buff (1982). This case is referred to as Var5. The size of the holes is equal to 0.15 for all these variants.

Presenting the results for Var3, it is convenient to show the enlarged picture of the magnetosphere. The main reason for this is a very small thickness of the shocked gas layer. This is due to the very efficient cooling of the gas behind the shock. 


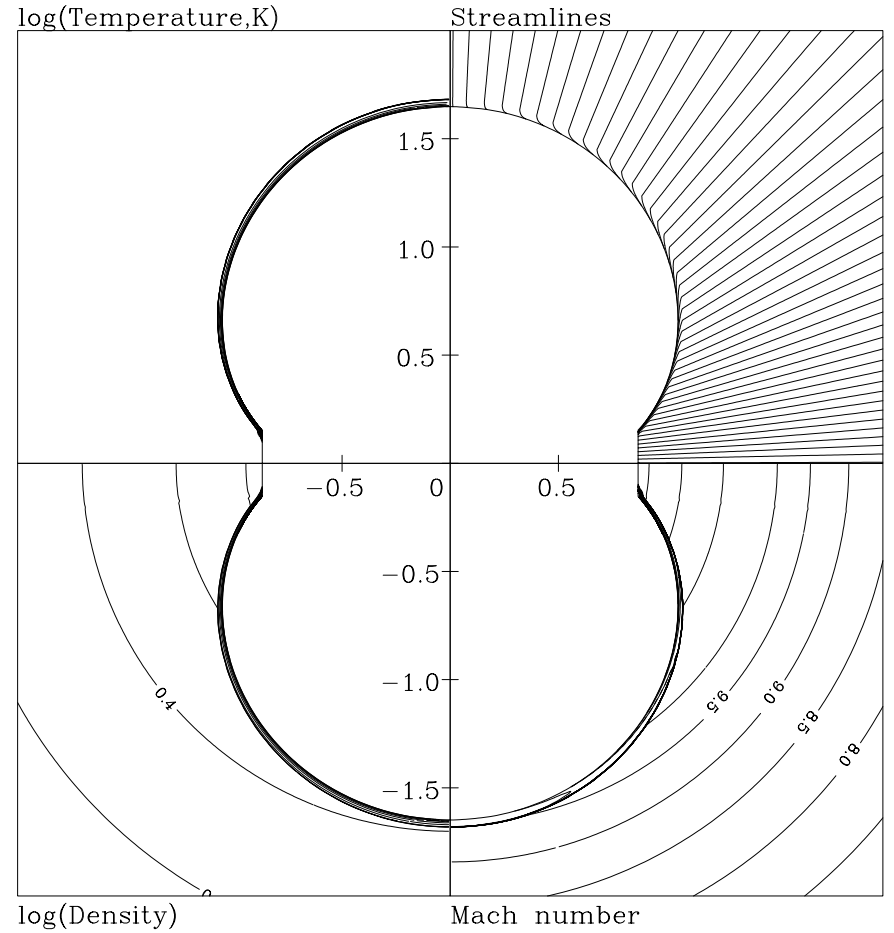

Fig. 11. Accretion pattern for the model Var3.

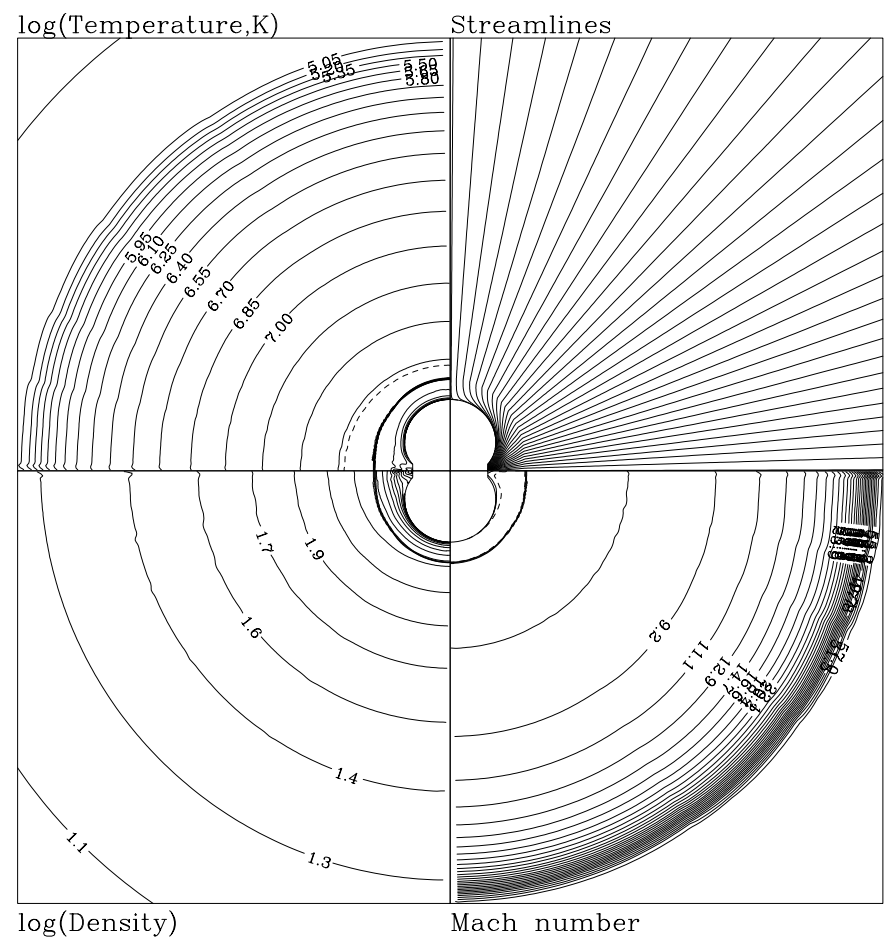

Fig. 12. Accretion pattern for Var4 with the cooling function by Cowie et al. (1981).

High compressivity of the matter leads to a large density rise in this layer, which results in the enhancement of the accretion ability of the star in the model under consideration. There is also no shock in front of the holes.

To understand the behavior of the the accreting gas, one should look at the one-dimensional plots shown in Fig. 14. We see that as we approach the star in the radial direction the plot

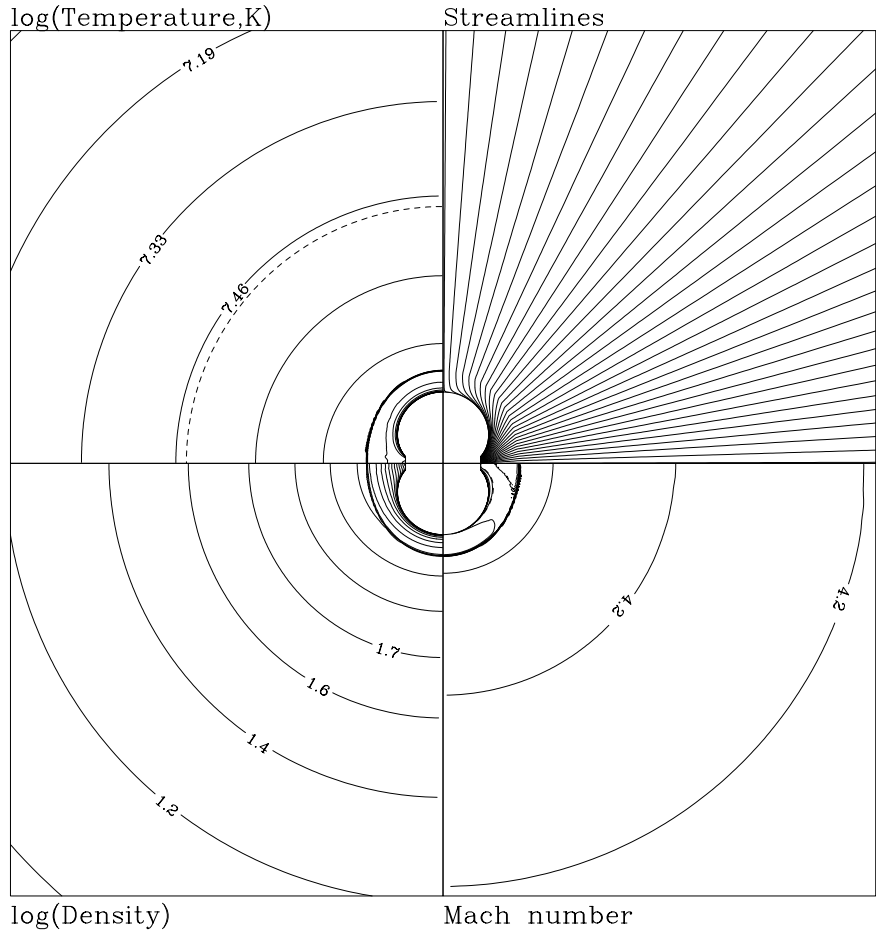

Fig. 13. Accretion pattern for Var5 with the cooling function by Stellingwerf \& Buff (1982).

of the corresponding velocity component becomes parallel to the free-fall velocity distribution indicated by the dashed line. This means that these velocities are proportional with a nearly constant coefficient. Of course, along the equatorial direction the velocity turns to zero, vanishing at the surface of the magnetosphere. A very interesting feature can be observed on the temperature and Mach number distributions. At a certain distance from the origin we see a jump-like increase in temperature, which is as high as nearly 2.5 orders of magnitude. This is not a shock, however, as seen from the behavior of the Mach number, which remains well above 1 . This behavior is somewhat similar to that observed by Chang \& Ostriker (1985), although, in contrast with their results, the flow remains continuous. This, in fact, means that preheated flows can be stabilized not only by gas dynamic shocks, as was suggested in the paper mentioned, but also by special shock-like structure manifesting a high temperature increase. We see that the gas in this case reaches the Compton temperature and remains in its vicinity everywhere except for the direct vicinity of the holes, where there is now a shock caused by the gas deceleration due to the boundary conditions. Note that the temperature increases monotonically from the outer boundary. The gradient is not extremely high, since radiative cooling takes some energy away. However, when we pass the maximum of the cooling function and it abruptly goes down, nothing can prevent the Compton heating.

As mentioned earlier, when the matter attains the Compton temperature, it tends to become nearly isothermal. This can be explained by strong cooling of the matter due to the inverse Compton effect (plus bremsstrahlung, of course, although it is substantially smaller than the former, especially at close 

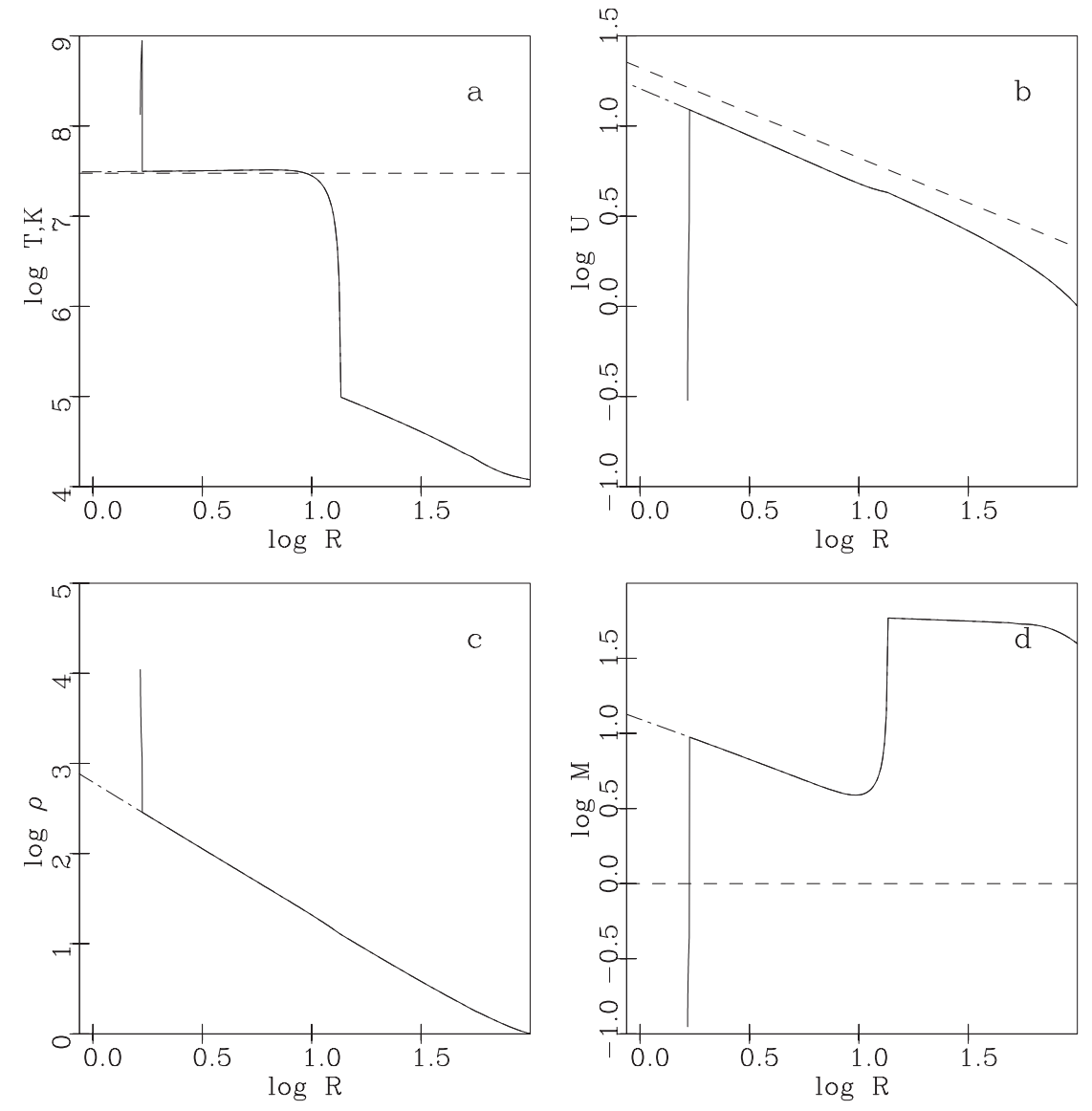

Fig. 14. Radial distributions of temperature a), radial velocity component b), density c), and Mach number d) along the $x$ - (solid lines) and $z$-axes (dash-and-dot lines). Dashed lines in figures a) and b) show the Compton temperature value and the free-fall velocity (Var3).
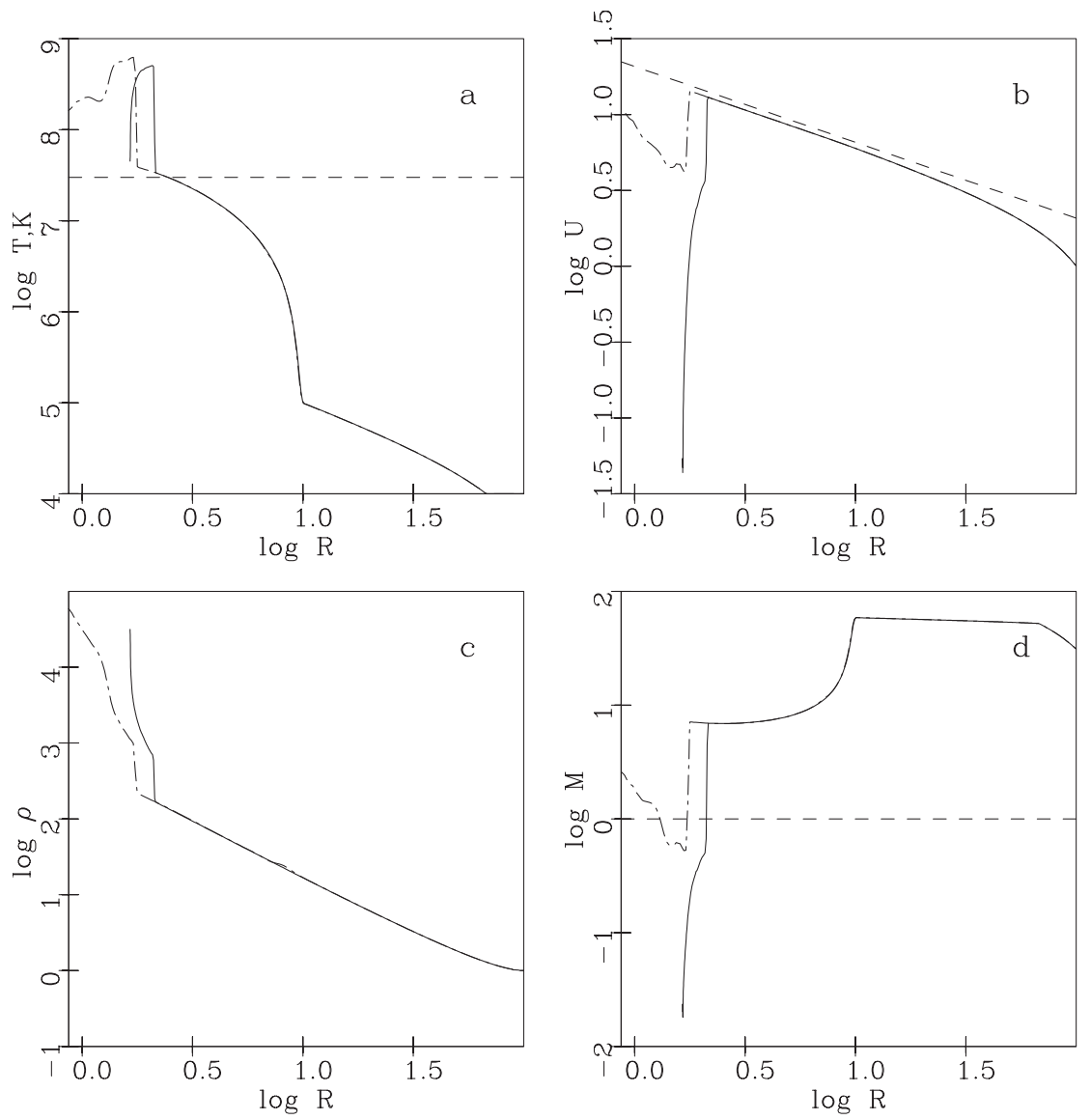

Fig. 15. Radial distributions of temperature a), radial velocity component b), density c), and Mach number d) along the $x$ - (solid lines) and $z$-axes (dash-and-dot lines). Dashed lines in figures a), b), and c) show the Compton temperature value, the free-fall velocity, and the $M=1$ value (Var4, the cooling function by Cowie et al. 1981). 

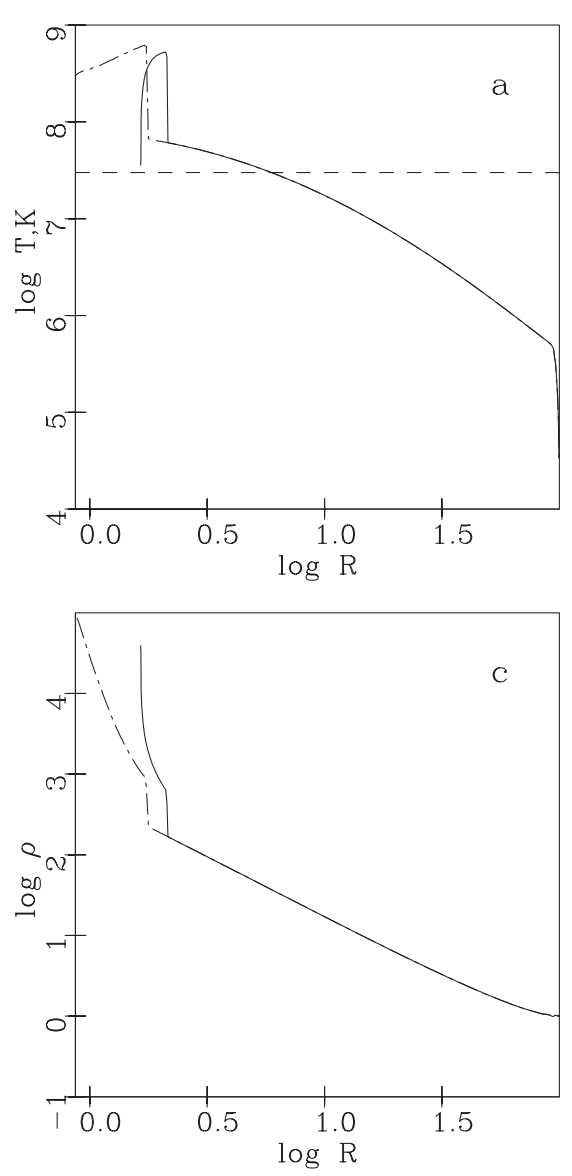
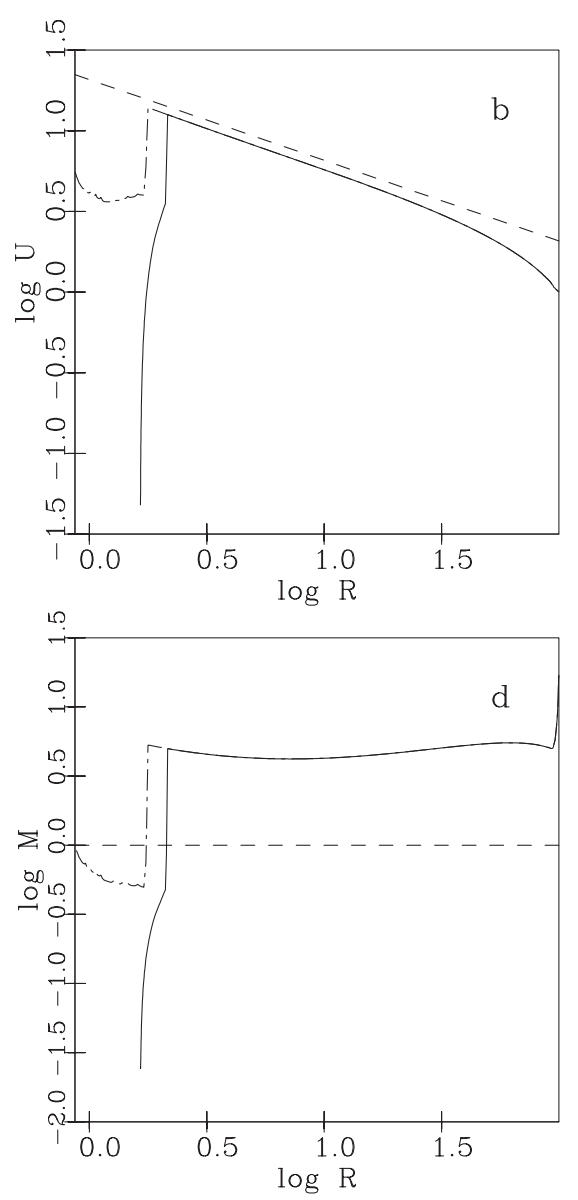

Fig. 16. Radial distributions of temperature a), radial velocity component $\mathbf{b})$, density $\mathbf{c})$, and Mach number d) along the $x$ - (solid lines) and $z$-axes (dash-and-dot lines). Dashed lines in figures a), b), and c) show the Compton temperature value, the free-fall velocity, and the $M=1$ value (Var5, the cooling function by Stellingwerf \& Buff 1982).

distances from the accretor) if the temperature exceeds the Compton temperature. If, vice versa, the temperature decreases below the Compton temperature, bremsstrahlung cannot compete with the Compton heating. Thus, the Compton effects, both direct and inverse, effectively attract the gas temperature to the Compton value.

Let us compare the results of Var4 and Var5. As seen from the temperature distributions, the action of the first cooling function is similar to that in Var3. The flow is isothermal near the outer boundary, then heating only slightly prevails over cooling, and the temperature gradient is positive, but not very steep. Note that in the one-dimensional plots we use logarithmic scales. When the cooling function goes down, we observe a sharp temperature increase that is accompanied by the increase in pressure, whereas the density remains smooth. As the gas approaches the accretor, it attains the Compton temperature right ahead of the shock on the equator, then heats crossing the shock and soon cools again due to the inverse Compton effect. Its behavior is substantially different at large distances if we use the second cooling function. In this case, cooling at the outer boundary is considerably lower and the gas temperature increases in its close vicinity. Then, the cooling mechanism switches on and the temperature gradient decreases. Since this function has no sharp negative gradient, there is no strong response while the gas passes from this branch of the cooling curve to the bremsstrahlung branch. The Compton temperature in Var5 is attained at greater distances from the star than with the cooling function by Cowie et al. (1981). This behavior is

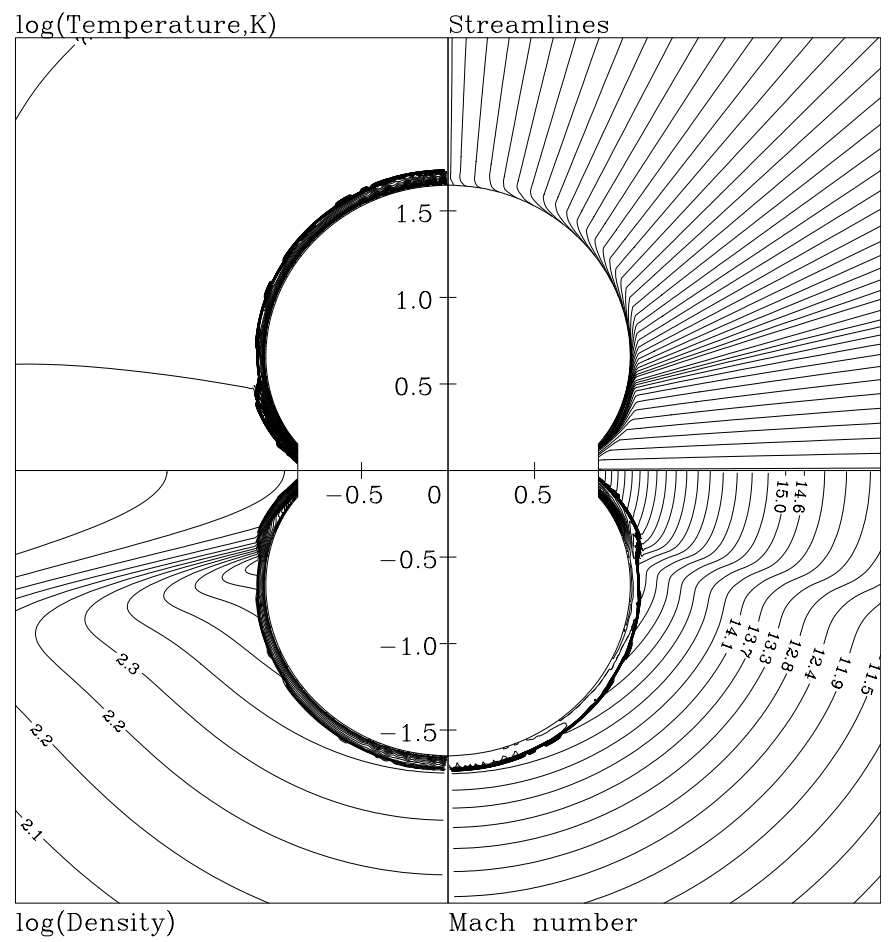

Fig. 17. Accretion pattern for Var1 with rotation $\left(S_{\mathrm{r}}=50\right)$.

clearly seen both on the one-dimensional plots and on the isoline charts. Although the gas temperature in the shock layer 

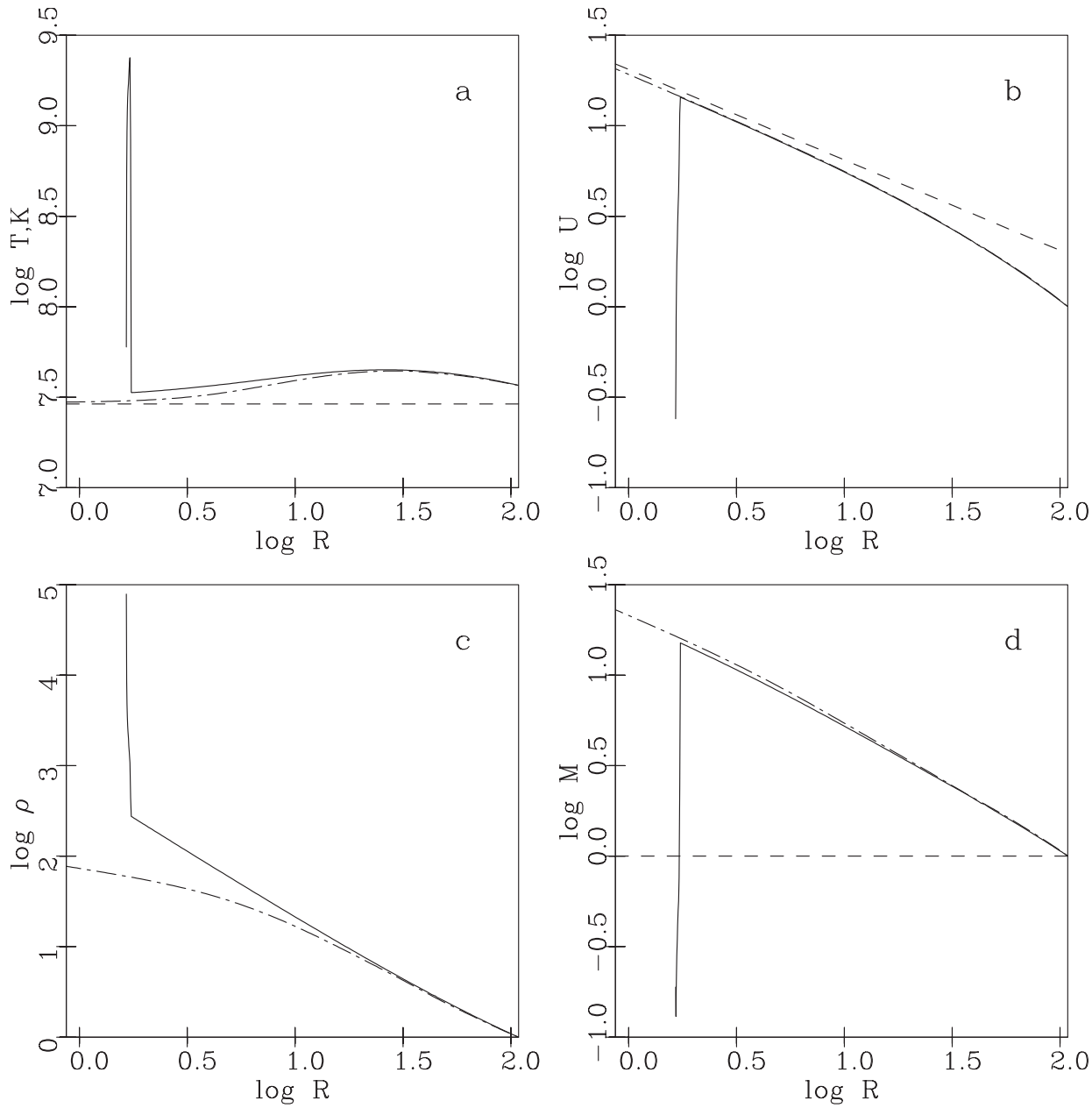

Fig. 18. Radial distributions of temperature a), radial velocity component $\mathbf{b}$ ), density c), and Mach number d) along the $x$ - (solid lines) and $z$-axes (dash-anddot lines). Dashed lines in figures $\mathbf{b}$ ) and c) show the free-fall velocity and the $M=1$ value (Var1 with rotation).

is very high, fast cooling of the gas inside it results in a fast rise of the density, thus enhancing the accretion ability of the model configuration.

It can be concluded that incorporating suitable physical radiative mechanisms allows one to substantially influence the accretion ability of the model magnetosphere. This implies that even narrow polar holes can accrete matter at a rate that is sufficiently high to allow for stationary solutions with standing shock waves ahead of the magnetosphere.

\section{Accretion of rotating gas on a stellar magnetosphere}

Suppose now that the gas at the outer boundary has nonzero angular momentum. In this case increasingly large centrifugal forces will act upon the matter as it approaches the surface of the magnetosphere. It is easy to predict that this force will hamper accretion (Paper I). Having this consideration in mind, we present the results of the numerical simulation of the accretion with the parameters at the outer boundary corresponding to Var 1 modified by rotation with the dimensionless parameter $S_{\mathrm{r}}=50$. The radius of the holes is equal to 0.15. In Figs. 17 and 18 we show the isoline patterns and one-dimensional plots along the $x$ - and $z$-axes. The flow ahead of the shock is obviously no longer spherically symmetric. One can see that the density in the vicinity of the axis of rotation is one order of magnitude less that in the case without rotation. The density distribution inside the shock layer becomes more uniform. Due to the action of the centrifugal force, the streamlines concentrate in the layers adjacent to the edges of the polar holes and accretion mainly occurs along the surface of a funnel wall.

If we decrease the parameter $S_{\mathrm{r}}$, the centrifugal force sooner or later increases to such an extent that outflows of rotating matter are observed along the polar axis. It is interesting that such outflows can occur without any magnetic field embedded into the matter, which is characteristic to the magnetic propeller accretion regime (Lovelace et al. 1999). This case is presented in Fig. 19, which corresponds to parameters at the outer boundary from Var4 and the parameter $S_{\mathrm{r}}=10$. A complicated flow structure which is observed in this case corresponds to a multiple interaction of discontinuities originating when the fluid particles moving straight along the axis of rotation interact with those moving inside a compressed shock layer and experiencing the action of an extremely strong centrifugal force that throws them back to the surface funnel wall. One can also see a transverse shock that decelerates the flow in the angular direction.

The results obtained show that the magnetosphere can be in the state of a quasi-equilibrium which is characterized by the presence of both accretion and outflow regimes. Numerical implementation of such flows encounters substantial difficulties 


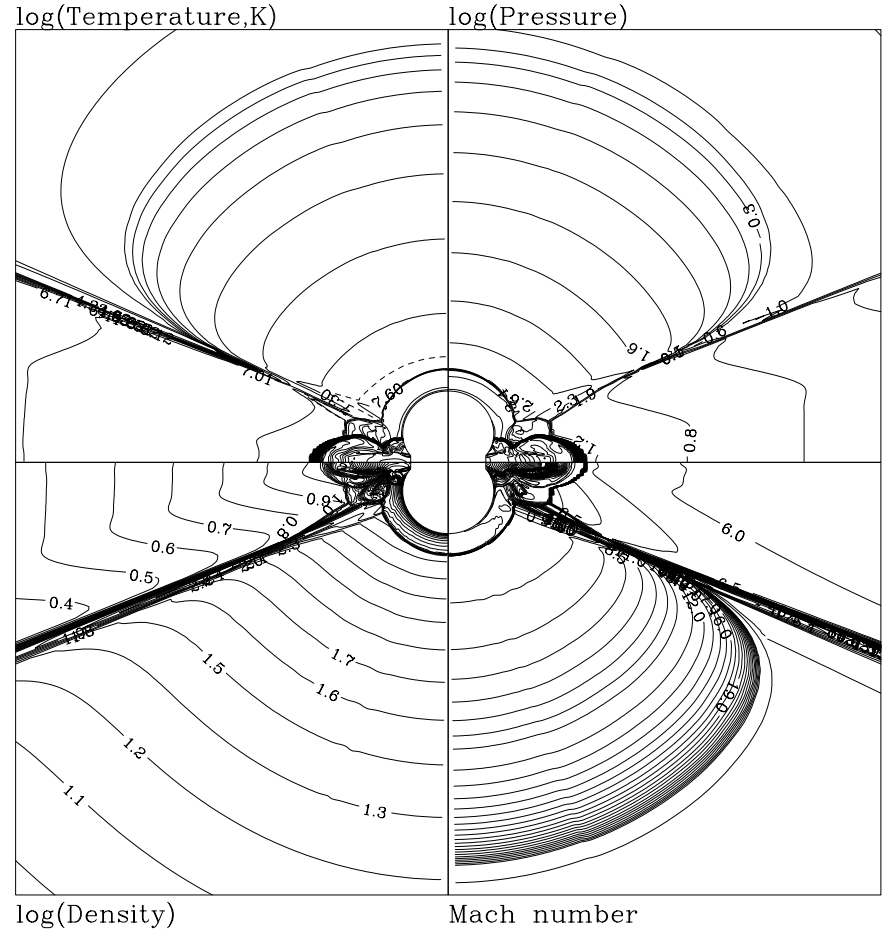

Fig. 19. The origin of an outflow in the model Var4 with strong rotation $\left(S_{\mathrm{r}}=10\right)$.

if the outflow reaches the outer boundary of the computational region.

\section{Discussion}

We studied the influence of radiative effects on the accretion patterns previously considered in Paper I in the polytropic approximation. The results can be subdivided into two groups. One of them deals with the classical Bondi-like accretion onto a gravitating center with or without rotation. It was shown that if we take the solution of BKB as the boundary condition at the outer spherical surface, a steady-state accretion pattern can be found that suffers no thermal instabilities in its supersonic part, which has actually been considered in this paper. The results obtained are in good agreement with the solution of the stationary problem by BKB. If the accreting matter possesses sufficient angular momentum at the outer boundary, a disk-like structure can be obtained that differs considerably from similar calculations based on the polytropic model. We found that the matter firstly gets compressed in the vicinity of the equatorial plane through transverse shocks. The gas temperature becomes nearly two orders of magnitude greater than the Compton temperature. One also finds that closer to the equatorial plane the gas becomes very dense, since the inverse Compton effect leads to a sharp decrease of temperature back to the values comparable with the Compton temperature. Although the "disk" tends to be comptonized, there exists a definite variation of temperature and density along its radial direction, which is caused by hydrodynamic effects resulting in the growth of the disk thickness in the direction toward the star. Our numerical example corresponds to the situation mentioned in Introduction which occurs in high-mass X-ray sources with long-periodic pulsars.
During accretion from a high-velocity stellar wind the angular velocity does not reach the Keplerian value at the Alfvén radius.

Another group of the presented results concerns quasispherical accretion onto the magnetosphere of a star. The model is based on the accretion scenario suggested by Arons \& Lea $(1976,1980)$, where the matter is accreted due to the disintegration of the initially impermeable, cusped magnetosphere caused by the Rayleigh-Taylor (interchange) instability. For the moment, we leave aside the study of the actual process of this penetration and admit the presence of an Alfvén surface (plasmasphere) which acts as a solid wall everywhere except for the polar holes, at which the accretion actually occurs. This model was used to resolve the question about the possibility of accretion patterns with a shock wave that lies in the near vicinity of the magnetosphere and does not propagate outward. If this happens, the matter heats up in the developing high-density shock layer and accretes in a way substantially differing from what can be expected in the case of a low-speed subsonic accretion. Such solutions have been obtained for various parameters at the outer boundary. As found in Paper I, efficient cooling of matter behind the shock favors accretion. This means that in this case even narrow holes can allow for high accretion rates. This conclusion is supported by the current paper, where we model radiative phenomena selfconsistently, remaining, of course, in the framework of an optically thin plasma. Moreover, higher accretion rates in our case resulted in more efficient cooling of the matter. It should be noted that the described accretion pattern can also represent an averaged picture in such cases where instabilities make the shock unsteady. The picture will remain qualitatively similar to that obtained here once this shock does not move away from the magnetospheric surface, as in the before-mentioned calculations by Toropin et al. (1999) that involved unjustifiably high electrical resistivity and, for certain regimes, in our calculations.

Any temperatures which are much above the Compton temperature can only be achieved by shock heating in such flows. In our calculations we encountered two different types of situations:

1. The flow ahead of the shock remains rather cool and the Mach number is rather large, typically 10-20. Therefore we obtain a very strong shock that produces the enormous heating observed in these models.

2. The flow ahead of the shock is heated up and the Mach number is correspondingly smaller (2-5). Then the shock will also be weaker. However, since the temperatures ahead of the shock are already very high, in these cases one gets similar temperatures behind the shock as in case 1. Very strong cooling immediately sets in behind this shock due to inverse Compton scattering. Therefore the region of extreme temperatures will always be very narrow (in some cases it is even difficult to resolve it numerically). The width of the high temperature region will obviously depend on the radial component of the flow velocity behind the shock. 
From the physical viewpoint it was interesting to determine how different cooling functions, which correspond to different ionization parameters and cosmic element abundances, can influence the overall accretion scenario. The choice of the cooling function, which is used in the energy equation, represents a large uncertainty in the entire investigation. It is quite clear that the radiation can be described by considering only optically thin losses. Then one can use the loss function presented by Cowie et al. (1981). This cooling function is obtained for purely collisional ionization and for a prescribed relative abundance of heavy elements. The curve shows an enormous peak around $10^{5} \mathrm{~K}$, which is entirely caused by resonance lines of weakly ionized heavy elements. This implies that exact values for the abundances of heavy elements are important. There is, however, an even more dramatic aspect related to the radiation from the central X-ray source: these X-rays can ionize heavy elements much beyond the collisional degree. This effect was pointed out by Buff \& McCray (1974) who proposed a modified cooling function which was meant as a rough approximation to the true situation. An analytical representation of that cooling curve has been given by Stellingwerf \& Buff (1982). In reality the situation is even more complicated. The degree of ionization of each species in a radiation field is determined by the ionization parameter $\xi=L /\left(n R^{2}\right)$, where $n$ represents the particle density. In our computational domain the parameter $\xi$ can vary over several orders of magnitude. The degrees of ionization for all the relevant species have been computed by Tarter et al. (1969) as functions of the parameter $\xi$. One sees from the curves presented in that paper that in the range $-1 \leq \log \xi \leq 3$ substantial changes in all these ionization degrees take place. If one now combines those results with the cooling curves of Sutherland \& Dopita (1993), which give the contributions of different elements to the total cooling curve, one could in principle determine the cooling function $\Lambda(T, \xi)$. This cooling function should then be used if one wants to calculate the exact temperature distribution in these accretion flows. Since this would require an enormous computational effort, we have not done this in the present paper. Instead we used the cooling curves of Stellingwerf \& Buff (1982) and Cowie et al. (1981) as lower and upper limits for the true cooling rate.

The difference between the flows for the two types of the cooling function is substantial at large distances from the magnetosphere of the star. Near the magnetosphere itself temperatures are very high for all cases and therefore cooling will be produced by bremsstrahlung. For this reason no large differences were found in this part of the computational domain. Moreover, one finds that for the very hottest parts of the flow the cooling is entirely due to the inverse Compton scattering. This implies that the innermost regions are insensitive to the choice of the cooling function and therefore our results for these regions will be quite general.

Concerning distant regions, we found that a very steep temperature increase can occur for the cooling function of Cowie et al. (1981). In contrast with the solutions by Chang \& Ostriker (1985), these jumps are not shocks. This can be explained by the fact that those authors used an oversimplified cooling function. Worth mentioning are also the results by
Nobili et al. (1991) who also could not obtain any solution with additional standing shocks at large distances from the accretor.

To check the applicability of the chosen model of an optically thin medium, we have calculated the optical depths along different radial directions between the $x$ - and $z$-axes for all considered variants. It has turned out that it never exceeded 0.9 (Var6) and was below 0.14 in Var3-Var5.

Summarizing, we would like to emphasize that radiative phenomena can strongly affect the accretion flow. On the other hand, incorporating the source terms responsible for these phenomena into the hydrodynamic code in the assumption of an optically thin medium very often requires certain caution, since these terms, being rapidly changing functions of temperature, density, and even the distance to the star, can make the mathematical problem stiff. This requires the application of specifically designed numerical techniques; see, e.g., LeVeque (1998), Gascón \& Corberán (2001) and references therein.

The problem of the quasi-spherical accretion onto a stellar magnetosphere was somewhat simplified in our investigation. First, we neglected magnetohydrodynamic phenomena that actually are responsible for the penetration of matter beneath the magnetosphere and the angular velocity distribution in the accreting matter behind the shock. We also assumed that the rotational axis of the star and/or of the accreting matter are aligned with the magnetospheric axis, which can hardly be expected in a general case. For example, all stellar X-ray sources that are observed as pulsars require a finite inclination angle between these axes. The three-dimensional MHD problem is rather complicated and will be the subject of further studies.

Acknowledgements. I.A.K. and N.V.P. are grateful to the Max-PlanckInstitut für Astrophysik in Garching bei München for hospitality. G.S.B.-K., I.A.K., and N.V.P. were partially supported by the Russian Foundation for Basic Research grant No. 02-01-00948.

\section{References}

Anzer, U., \& Börner, G. 1995, A\&A, 299, 62

Arons, J., \& Lea, S. M. 1976, ApJ, 207, 914

Arons, J., \& Lea, S. M. 1980, ApJ, 235, 1016

Baushev, A. V., \& Bisnovatyi-Kogan, G. S. 1999, Astron. Rep., 43, 241

Bisnovatyi-Kogan, G. S. 1991, A\&A, 245, 528

Bisnovatyi-Kogan, G. S., \& Blinnikov, S. I. 1980, MNRAS, 191, 711

Bisnovatyi-Kogan, G. S., \& Pogorelov, N. V. 1997, Astron. Astrophys. Trans., 12, 263

Bond, J. R., Centrella, J., Szalay, A. S., \& Wilson, J. R. 1984, MNRAS, 210, 215

Bondi, H. 1952, MNRAS, 112, 195

Börner, G., Hayakawa, S., Nagase, F., \& Anzer, U. 1987, A\&A, 182, 63

Buff, J., \& McCray, R. 1974, ApJ, 189, 147

Chang, K. M., \& Ostriker, J. P. 1985, ApJ, 288, 428

Chen, X., Taam, R. E., Abramovicz, M. A., \& Igumenshchev, I. V. 1997, MNRAS, 285, 439

Cowie, L. L., Ostriker, J. P., \& Stark, A. A. 1978, ApJ, 226, 1041

Cowie, L. L., McKee, C. F., \& Ostriker, J. P. 1981, ApJ, 247, 908

Cox, D. P., \& Tucker, W. H. 1964, ApJ, 157, 1157

Elsner, R. F., \& Lamb, F. K. 1976 ApJ, 215, 897

Gascón, Ll., \& Corberán, J. M. 2001, J. Comput. Phys., 172, 261 
Hutchings, R. M, \& Thomas, P. A. 2000, MNRAS, 319, 721

Igumenshchev, I. V., Illarionov, I. F., \& Kompaneets, D. A. 1993, MNRAS, 260, 727

Illarionov, I. F., \& Kompaneets, D. A. 1990, MNRAS, 247, 219

Illarionov, I. F., \& Sunyaev R. A. 1975, A\&A, 39, 185

Ivanov, I. E., \& Kryukov, I. A. 1996, Matematicheskoe Modelirovanie [Mathematical Modeling], 8, No. 6, 47 [in Russian]

Ivanov, I. E., Kryukov, I. A., \& Pogorelov, N. V. 1999, in Proc. 8th Meshing Roundtable, Lake Tahoe, 1999, Sandia Report SAND99-2288, Albuquerque, NM, 313

Kazhdan, Ya. M., \& Murzina, M. 1994, MNRAS, 270, 351

Krolik, J. H., \& London, R. A. 1983, A\&A, 267, 18

Kryukov, I. A., Pogorelov, N. V., Bisnovatyi-Kogan, G. S., Anzer, U., \& Börner, G. 2000, A\&A, 364, 901

Kulikovskii, A. G., Pogorelov, N. V., \& Semenov, A. Yu. 2000, Mathematical Aspects of Numerical Solution of Hyperbolic Systems (CRC Press/Chapman \& Hall, Boca Raton, FL/London, U.K.)

Lipunov, V. M. 1992, Astrophysics of Neutron Stars (Berlin: Springer)

LeVeque, R. J. 1998, J. Comput. Phys., 146, 346

Levich, E. V., \& Sunyaev, R. A. 1971, AZh, 15, 363

Lovelace, R. V. E., Romanova, M. M., \& Bisnovatyi-Kogan, G. S. 1999, ApJ, 514, 368
Molteni, D., Tóth, G., \& Kuznetsov, O. A. 1999, ApJ, 516, 411

Nagase, F. 1989, PASJ, 41, 1

Nobili, L., Turola, R., \& Zampieri, L. 1991, ApJ, 383, 250

Ostriker, J. P., McCray, R., Weaver, R., \& Yahil, A. 1976, ApJ, 208, L61

Park, M.-G. 1990, ApJ, 354, 64

Pogorelov, N. V., \& Matsuda, T. 1998, in CFD Rev. 1998, ed. M. Hafez, \& K. Oshima (Singapore: World Scientific), 2, 932

Pogorelov, N. V., \& Semenov, A. Yu. 1997, A\&A, 321, 330

Raymond, J. C., Cox, D. P., \& Smith, B. W. 1976, ApJ, 204, 290

Ruffert, M. 1994, ApJ, 427, 342

Ruffert, M., \& Arnett, D. 1994, ApJ, 427, 351

Scott, D. M., Leahy, D. A., \& Wilson, R. B. 2000, ApJ, 539, 392

Shapiro, S. L., \& Lightman, A. 1976, ApJ, 204, 555

Sheffer, E., Kopaeva, I. F., Averintsev, M. B., et al. 1992, Soviet Ast., 36,41

Shvarzman, V. F. 1970, AZh, 47, 824

Stellingwerf, R. F., \& Buff, J. 1982, ApJ, 260, 755

Sutherland, R. S., \& Dopita, M. A. 1993, ApJS, 88, 253

Tarter, C. B., Tucker, W. H., \& Salpeter, E. E. 1969, ApJ, 156, 943

Toropin, Yu. M., Toropina, O. D., Savelyev, V. V., et al. 1999, ApJ, 517, 906 